\title{
Singly- and Triply-Linked Magnetic Porphyrin Lanthanide Arrays
}

\author{
Jeff M. Van Raden, ${ }^{\ddagger[a]}$ Dimitris I. Alexandropoulos, ${ }^{[[b]}$ Michael Slota, ${ }^{;[b]}$ Simen Sopp, ${ }^{[b]}$ Taisuke Matsuno, ${ }^{[c]}$ Am- \\ ber L. Thompson, ${ }^{[a]}$ Hiroyuki Isobe, ${ }^{[c]}$ Harry L. Anderson, ${ }^{*[a]}$ and Lapo Bogani ${ }^{*[b]}$
}

\author{
[a] Department of Chemistry, University of Oxford, Chemistry Research Laboratory, Oxford, OX1 3TA, UK \\ [b] Department of Materials, University of Oxford, OX1 3PH, Oxford, UK
}

[c] Department of Chemistry, The University of Tokyo, Tokyo 113-0033, Japan

KEYWORDS. porphyrin, lanthanide, dimer, chiral, magnetic, electronic

\begin{abstract}
The introduction of paramagnetic metal centers into a conjugated $\pi$-system is a promising approach towards engineering spintronic materials. Here, we report an investigation of two types of spin-bearing dysprosium(III) and gadolinium(III) porphyrin dimers: singly meso-meso-linked dimers with twisted conformations and planar edge-fused $\beta$,meso, $\beta$-linked tapes. The rareearth spin centers sit out of the plane of the porphyrin, so that the singly linked dimers are chiral, and their enantiomers can be resolved, whereas the edge-fused tape complexes can be separated into syn and anti stereoisomers. We compare the crystal structures, UV-vis-NIR absorption spectra, electrochemistry, EPR spectroscopy and magnetic behavior of these complexes. Low temperature SQUID magnetometry measurements reveal intramolecular antiferromagnetic exchange coupling between the Gd ${ }^{\text {III }}$ centers in the edge-fused dimers (syn isomer: $J=-51 \pm 2 \mathrm{MHz}$; anti isomer: $J=-19 \pm 3 \mathrm{MHz}$ ), whereas no exchange coupling is detected in the singly-linked twisted complex. The phase memory times, $T_{\mathrm{m}}$, are in the range $8-10 \mu \mathrm{s}$ at $3 \mathrm{~K}$, which is long enough to test quantum computational schemes using microwave pulses. The syn and anti $\mathrm{Dy}_{2}$ edge-fused tapes both exhibit single molecule magnetic hysteresis cycles at temperatures below $0.5 \mathrm{~K}$ with slow magnetization dynamics.
\end{abstract}

\section{Introduction}

Graphene-like materials with extensive $\pi$-delocalization exhibit remarkable electronic and physical properties. ${ }^{1}$ One poorly explored aspect is the injection of spin into the delocalized states of a $\pi$-conjugated backbone. ${ }^{2}$ The deposition of single-molecule magnets (SMMs) on graphene revealed not only sizeable spin-electron interaction, but also the possibility of driving the spin dynamics into fully quantum regimes, such as Villain's tunneling region. ${ }^{3}$ On the other hand, we lack fundamental information about how to engineer such interactions: How are spin interactions transmitted along a $\pi$-conjugated plane? How do they behave when spins are on the same or opposite sides of the plane? What happens when a twist blocks $\pi$-conjugation? And how does conjugation influence the spin dynamics?

Previously, attempts have been made to address these questions by depositing metals and magnetic molecules on graphene, ${ }^{4}$ but this results in random molecular placement. The chemical doping of graphene yields structures that are poorly defined at the atomic level, hampering the elucidation of structure-property relationships. For example, although the edges of graphene nanoribbons had long been proposed to exhibit ferromagnetism, spin-filtering capabilities, ${ }^{5}$ and quantum-coherence features, ${ }^{6}$ only the advent of molecular graphene nanoribbons with atomically precise structures enabled experimental investigation of magnetic edge states. ${ }^{7}$ Molecular metal coordination complexes with $\pi$-conjugated backbones and a few spins offer unexplored opportunities to address these issues, by providing spin-functionalized conjugated frameworks, ${ }^{8}$ with atomic-level control, enabling spin-spin interactions to be rationalized, and the best frameworks selected.

$\mathrm{Ln}^{\text {III }}$-based single-molecule magnet (SMM) $)^{9}$ can offer high blocking temperatures and ultra-hard magnetic behavior. ${ }^{10}$ Moreover, $\mathrm{Ln}^{\text {III }}$ complexes afford an extreme level of tuning of the magnetic properties, by changing the rare-earth, without altering the chemistry or the structural features. Changing the $\mathrm{Ln}^{\mathrm{III}}$ cation provides control over the spin-orbit coupling, and thus the interplay of electronic and spin degrees of freedom in the conjugated backbone, and facilitates elucidation of both the SMM behavior and coherent states. Metalation of porphyrins with $\mathrm{Ln}^{\mathrm{III}}$ cations is thus an excellent strategy to introduce spin into $\pi$-conjugated materials, and to investigate magnetic coupling through large aromatic $\pi$-systems. Previously, we have shown that butadiyne-linked lanthanide porphyrin dimers exhibit slow magnetic relaxation below 10 kelvin under a static magnetic field, and that they provide the necessary elements for construction of a single-molecule spin valve. ${ }^{11}$ Mesosingly-linked porphyrin oligomers ${ }^{12}$ (Figure 1a) and $\beta$, meso, $\beta$ edge-fused porphyrin tapes ${ }^{13}$ (Figure $1 \mathrm{~b}$ ) can be regarded as yin and yang structures: their connectivity is similar but they display opposite types of electronic coupling. Single-linked chains are highly twisted, with neighboring porphyrins almost orthogonal, preventing orbital overlap, but there is strong through-space exciton coupling between the porphyrins, and the chains behave as photonic wires. ${ }^{14}$ In contrast, the fused tapes have flat $\pi$-systems with strong $\pi$-conjugation; their $\pi-\pi^{*}$ energy gaps fall steeply with increasing oligomer length, ${ }^{13,15}$ and their single-molecule conductances are almost independent of length. ${ }^{16}$ Diamagnetic porphyrin oligomers, containing zinc(II) or nickel(II) cations, have been 
thoroughly investigated, but there have been few studies of the magnetic properties of singly-linked oligomers and triply-linked tapes hosting paramagnetic metal centers. ${ }^{17,18}$ Here we investigate both singly-linked and fused porphyrin dimers with dysprosium(III) or gadolinium(III) centers, as models for longer oligomer with many lanthanide metal cations. In contrast to metals such as $\mathrm{Zn}^{\mathrm{II}}, \mathrm{Cu}^{\mathrm{II}}$ and $\mathrm{Ni}^{\mathrm{II}}$, which sit in the plane of the porphyrin, $\mathrm{Dy}{ }^{\mathrm{III}}$ and $\mathrm{Gd}^{\mathrm{III}}$ sit out of plane, leading to interesting issues of stereochemistry. We investigate axially chiral singly-linked dinuclear complexes (Figure 1c) and triply-linked stereoisomeric dinulear metal complexes: the syn $(Z)$ isomer, in which both lanthanide metal centers are on the same face of the $\pi$-system and the anti $(E)$ isomer, in which the metals are on opposite faces (Figure 1d).

(a) singly-linked oligomers

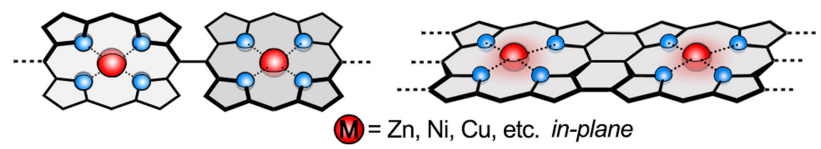

(c) singly-linked lanthanide arrays

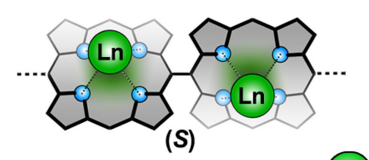

Ln $=$ Dy or Gd out-of-plane

(d) triply-linked lanthanide arrays
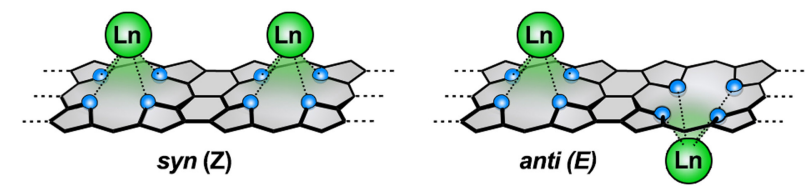

Figure 1. Cartoon representations of (a) singly- and (b) triply-linked porphyrin oligomers; (c) $\mathrm{Ln}^{\mathrm{III}}$-derived axially chiral singly-linked porphyrin arrays; (d) and triply-linked porphyrin arrays.

In this study, we investigate the structure-property relations in porphyrin oligomers coordinating $\mathrm{Ln}^{\mathrm{III}}$ centers. We test the SMM behavior and the quantum coherence times, while varying the $\mathrm{Ln}^{\mathrm{III}}$ metal cation and the stereochemistry; for example, metal ions sitting on the same or opposite sides of the $\pi$-conjugated plane give distinctively different coherence and hysteresis. We compare the properties of a lanthanide porphyrin monomer, P1 $\cdot \mathbf{L n}$ (Scheme 1; $\mathrm{Ln}=\mathrm{Dy}$ or $\mathrm{Gd}$ ) with two types of dimer: $\boldsymbol{s}-\mathbf{P} 2 \cdot \mathbf{L n}_{\mathbf{2}}$ (as two enantiomers) and $\mathbf{f} \mathbf{P} \mathbf{2} \cdot \mathbf{L n}_{2}$ (as two diastereomers, $E$ and Z, Scheme 2 ). In all these complexes, the lanthanide metal centers are protected by the Kläui capping ligand. ${ }^{11,19,20}$ This anionic cap is an important part of the molecular design because it results in neutral complexes that are soluble in nonpolar organic solvents, kinetically stable and easy to purify by chromatography on silica. ${ }^{11,19}$ The diamagnetic Co ${ }^{\text {III }}$ cation of this capping group does not significantly influence the magnetic properties. The crystal structures of $s-\mathrm{P} 2 \cdot \mathrm{Dy}_{2}, f-\mathrm{P} 2-Z \cdot \mathrm{Dy}_{2}$ and $\boldsymbol{f} \mathbf{P} 2-\boldsymbol{E} \cdot \mathrm{Dy}_{2}$ confirm their identities, while UV-vis-NIR absorption spectra and electrochemical measurements reveal differences in electronic structure. The impact of the stereochemistry and connectivities on the static and dynamic magnetic properties have been tested, including the coherence properties of porphyrin dimers bearing $\mathrm{Gd}^{\mathrm{III}}$ centers, $\boldsymbol{s}-\mathrm{P} 2 \cdot \mathrm{Gd}_{2}, \boldsymbol{f} \mathbf{P} \mathbf{2}-\boldsymbol{Z} \cdot \mathrm{Gd}_{2}$ and $\boldsymbol{f} \mathbf{P} \mathbf{2}-\boldsymbol{E} \cdot \mathrm{Gd}_{2}$.

\section{Results and Discussion}

Synthesis. The reference porphyrin monomer dysprosium and gadolinium complexes, $\mathbf{P} 1 \cdot \mathrm{Dy}$ and $\mathrm{P} 1 \cdot \mathrm{Gd}$, and the dimers $s$ $\mathrm{P} 2 \cdot \mathrm{Dy}_{2}, f-\mathrm{P} 2-Z \cdot \mathrm{Dy}_{2}, f-\mathrm{P} 2-E \cdot \mathrm{Dy}_{2}, s-\mathrm{P} 2 \cdot \mathrm{Gd}_{2}, f-\mathrm{P} 2-Z \cdot \mathrm{Gd}_{2}$ and $f-\mathrm{P} 2-$ $E \cdot \mathrm{Gd}_{2}$ were synthesized from the corresponding free-base porphyrins by treatment with the lanthanide(III) chlorides in diphenyl ether at $250{ }^{\circ} \mathrm{C}$, as shown in Schemes 1 and 2..$^{11,20}$ Use of sulfolane as a cosolvent was found to accelerate metalation of the porphyrin monomer, $\mathbf{P 1} \cdot \mathrm{H}_{2}{ }^{21}$ reducing the reaction time to 45 minutes, which is beneficial because long reaction times at this temperature result in decomposition. After insertion of the metal, Kläui's anionic capping ligand $\left(\mathrm{L}^{-}\right)$was coordinated to the metalloporphyrins at room temperature. ${ }^{19}$

\section{Scheme 1. Synthesis of metalloporphyrin monomer $\mathrm{P} 1 \cdot \mathrm{M}_{2}{ }^{\mathrm{a}}$}
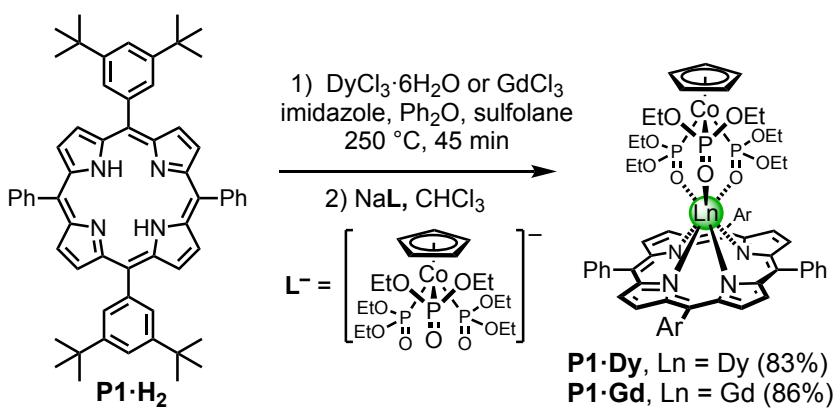

${ }^{a} \operatorname{Ar}=3,5-\operatorname{di}(t$-butyl $)$ phenyl.

In contrast to $\mathbf{P} \mathbf{1} \cdot \mathrm{H}_{2}$, use of sulfolane significantly reduced the yield for metalation of the dimers $s-\mathbf{P} 2 \cdot \mathrm{H}_{4}$ and $\mathbf{f}-\mathbf{P} 2 \cdot \mathrm{H}_{4}$. Consequently, sulfolane was omitted (Scheme 2 ) and these dimers required longer reaction times. As for $\mathbf{P 1} \cdot \mathbf{L n}$, the singly-linked products, $s$-P2 $\cdot \mathbf{L n}_{2}$, were purified by silica gel chromatography. The enantiomers were then resolved by chiral HPLC using a SUMICHIRAL column as discussed below. Separation of the syn and anti isomers ( $\boldsymbol{f}-\mathbf{P} \mathbf{2}-\boldsymbol{Z} \cdot \mathbf{L n}_{\mathbf{2}}$ and $\boldsymbol{f} \mathbf{- P 2}-\boldsymbol{E} \cdot \mathbf{L n}_{2}$, respectively) was accomplished by silica gel chromatography, followed by crystallization. As expected, the syn isomers are more polar than the anti isomers, leading to a lower chromatographic mobility on silica. Analysis of the crude reaction mixture of the Dy ${ }^{\mathrm{III}}$ reaction via gelpermeation chromatography (GPC) revealed an approximate 2:1 ratio of $Z: E$ isomers, and this observation is reflected in the isolated yields. It is surprising that the syn isomers predominate in these reactions, and it suggests an attractive interaction between the two metal centers.

We also synthesized $f-\mathrm{P} 2-E \cdot \mathrm{Dy}_{2}$ and $f-\mathrm{P} 2-E \cdot \mathrm{Gd}_{2}$ in high yield by Scholl reaction of $s-\mathrm{P} 2 \cdot \mathrm{Dy}_{2}$ and $s-\mathrm{P} 2 \cdot \mathrm{Dy}_{2}$, respectively, using phenyliodine(III) bis(trifluoroacetate) (PIFA), followed by work-up with sodium borohydride (Scheme 2; Route 2). ${ }^{22}$ It is surprising that the organometallic Kläui ligand survives these strongly oxidizing conditions and that this reaction proceeds so efficiently. It is also surprising that oxidation of $s-\mathbf{P 2} \cdot \mathbf{L n}_{2}$ gives exclusively $f \mathbf{P 2}$ $E \cdot \mathbf{L n}_{2}$, without forming detectable amounts of the $Z$-isomer, whereas metalation of $\mathbf{f} \mathbf{P} 2 \cdot \mathbf{H}_{4}$ gives predominantly the $Z$-isomer. The explanation for this difference in stereochemical outcome is probably that metalation occurs before the bulky Kläui ligand has been installed, when there is no steric clash between the metal centers, whereas the Scholl reaction (Route 2) is carried out with the bulky capping ligands in place. The efficient and highly stereoselective formation of $\boldsymbol{f}-\mathrm{P} 2-\boldsymbol{E} \cdot \mathrm{Dy}_{2}$ from $\boldsymbol{s}-\mathbf{P} 2 \cdot \mathrm{Dy}_{2}$ suggests that this 
route could be extended to prepare lanthanide complexes of long porphyrin oligomer tapes without forming mixtures of stereoisomers.

Scheme 2. Synthesis of the metalloporphyrin dimers (a) racemic $s$ $\mathrm{P} 2 \cdot \mathrm{M}_{2}$ and (b) $f-\mathrm{P} 2-Z \cdot \mathrm{M}_{2}$ and $f-\mathrm{P} 2-E \cdot \mathrm{M}_{2}{ }^{\mathrm{a}}$

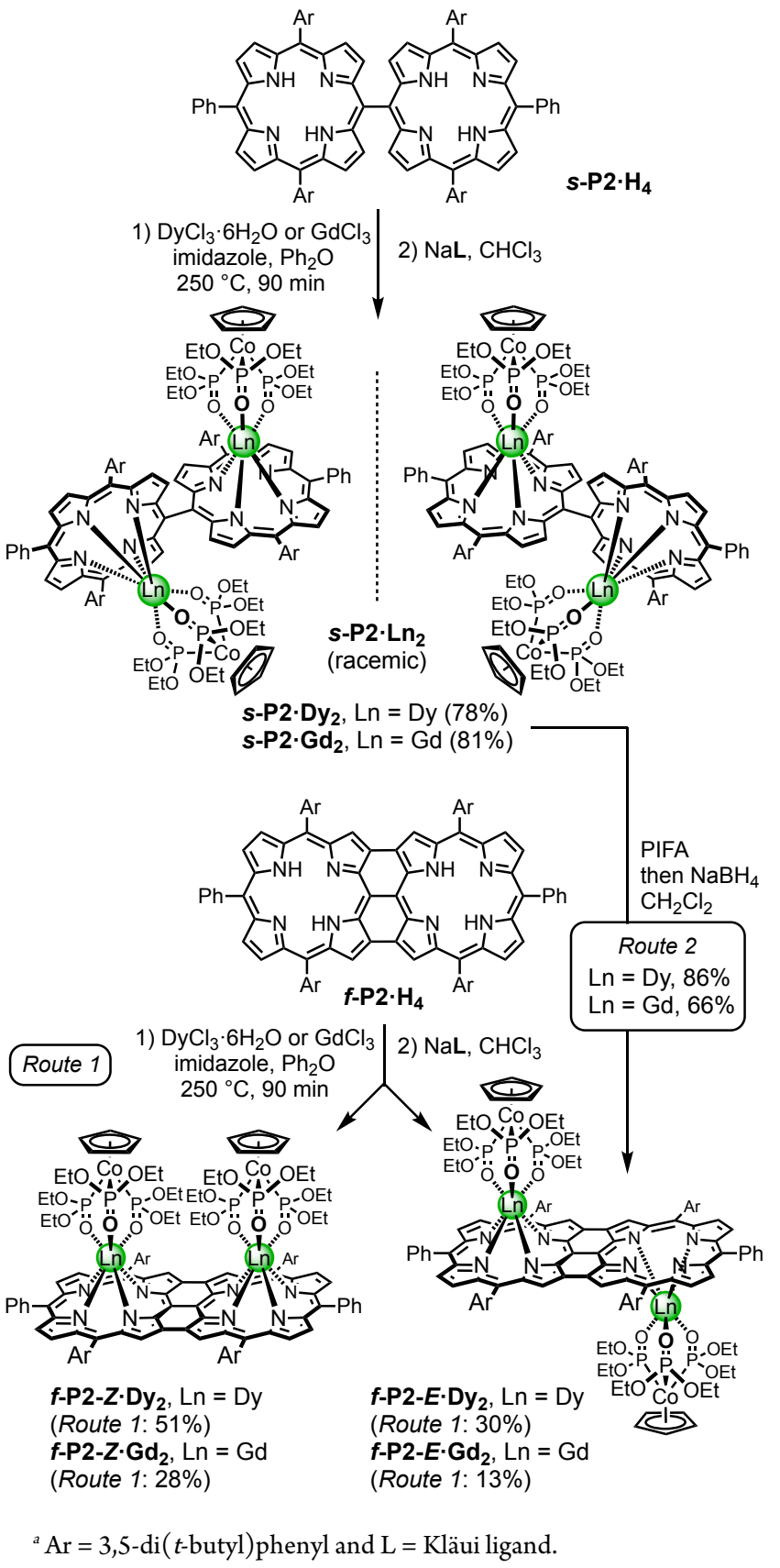

X-Ray Crystallography. ${ }^{23}$ Single crystals of $s-\mathbf{P} 2 \cdot \mathrm{Dy}_{2}$ and $s-\mathrm{P2} \cdot \mathrm{Gd}_{2}$ suitable for $\mathrm{X}$-ray diffraction were grown via liquid-liquid diffusion of methanol into chloroform solutions. The structures of these Dy and Gd complexes were found to be isomorphous and isostructural in the crystalline solid state, so we only discuss that of $s-\mathbf{P 2} \cdot \mathbf{D y}_{2}$ here (Figure 2). The structure of $\boldsymbol{s}-\mathbf{P} \mathbf{2} \cdot \mathbf{G d}_{2}$ is included in the Supporting Information (CIF). The capped $\mathrm{Ln}^{\mathrm{III}}$ metal cation coordinates to one face of the porphyrin, making the singly-linked porphyrin dimers axially chiral, and they crystallize as racemates. Both the Kläui capping groups in this structure are rotationally disordered, reflecting a shallow energy profile for rotation about the Dy-
Co axis. The angle between the planes defined by the four nitrogen atoms of the two porphyrins is $62.77(17)^{\circ}$, which is smaller than might be expected. For example, the corresponding angles in the crystal structures of two similar singly-linked zinc porphyrin dimer units are $69^{\circ}$ and $72^{\circ} \cdot{ }^{12 c, 24}$ The smaller torsional angle in $\boldsymbol{s}-\mathbf{P 2} \cdot \mathbf{D y _ { 2 }}$ may be a consequence of the steric bulk of the lanthanide capping group, which would clash with the aryl group of the neighboring porphyrin if the two porphyrins were orthogonal. The distances of the Dy ${ }^{\text {III }}$ centers from the mean planes of the porphyrins (defined by the four nitrogen atoms) are 1.22(1) and 1.23(1) $\AA$, similar to those reported in related Dy ${ }^{\text {III }}$ porphyrin complexes. ${ }^{11,20}$ The intramolecular Dy ${ }^{\cdots}$ Dy distance is 8.9451 (4) $\AA$, compared with 8.30 and $8.41 \AA$ in the analogous zinc complexes. ${ }^{12 c, 24}$ The Co-Dy vectors are almost perpendicular to the mean planes of nitrogen atoms of each porphyrin $\left(\theta=86.68(12)^{\circ}\right.$ and $89.01(14)^{\circ}$, Figure 2$)$.
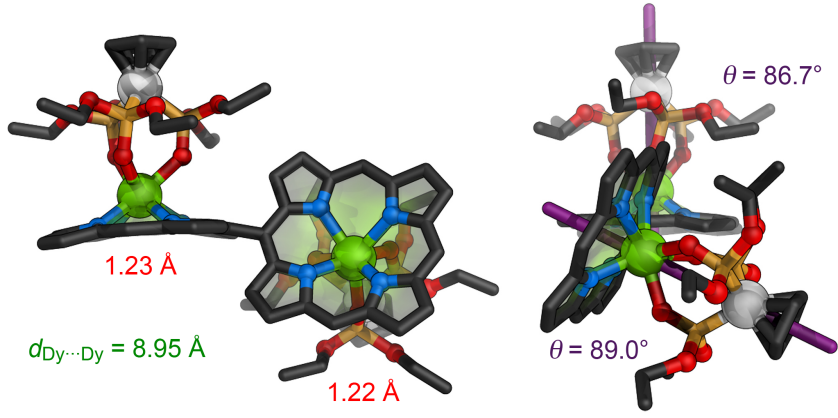

Figure 2. Crystal structure of $\boldsymbol{s}-\mathbf{P} \mathbf{2} \cdot \mathrm{Dy}_{2}$ from X-ray diffraction studies showing side (left) and axial views (right). Solvent molecules, hydrogen atoms, aryl groups and minor components of disorder are omitted for clarity. The violet lines indicate the Co-Dy vectors. The distances of the Dy atoms from the mean planes of the four nitrogen atoms are shown in red and the angle $\theta$ of the Co-Dy vectors to the planes for the four nitrogen atoms are shown in purple.

Single crystals of the edge-fused lanthanide porphyrin dimers were grown via liquid-liquid diffusion, by layering ethyl acetate or methanol over chloroform solutions of $\boldsymbol{f}-\mathrm{P} 2-\boldsymbol{Z} \cdot \mathrm{D} \mathbf{y}_{2}$ or $\boldsymbol{f}-\mathrm{P} 2-\boldsymbol{E} \cdot \mathrm{Dy}_{2}$, respectively. Both structures (Figure 3 ) have half a porphyrin dimer molecule in the asymmetric unit: the two halves of the $\mathbf{f} \mathbf{P 2}-\boldsymbol{Z} \cdot \mathbf{D} \mathbf{y}_{2}$ molecule are related by a crystallographic mirror plane, which lies in the $\mathrm{Dy}_{2} \mathrm{Co}_{2}$ plane, whereas the $f-\mathrm{P} 2-\boldsymbol{E} \cdot \mathrm{Dy}_{2}$ molecule occupies a position on an inversion center. The intramolecular Dy $\cdots$ Dy distances are 8.5561(9) $\AA$ in $\mathbf{f} \mathbf{P} 2-\boldsymbol{Z} \cdot \mathbf{D y}_{2}$ and 8.9371(9) $4 \AA$ in $\boldsymbol{f} \mathbf{P 2}$ $\boldsymbol{E} \cdot \mathrm{Dy}_{2}$ (Figure 3a,b). This compares with a $\mathrm{Zn} \cdots \mathrm{Zn}$ distance of 8.45 A in closely related complex of the type $\mathbf{f} \mathbf{P} 2 \cdot \mathrm{Zn}_{2} \cdot{ }^{25}$ The distances of the $\mathrm{Dy}^{\mathrm{III}}$ centers from the mean planes of the porphyrins (defined by the four nitrogen atoms) are similar to those in $\boldsymbol{s}-\mathbf{P} 2 \cdot \mathbf{D y}_{2}$ (1.2169(6) and 1.2671(5) $\AA$ in $f-P 2-Z \cdot D_{2}$ and 1.2164(6) $\AA$ in $f-$ $\left.\mathrm{P} 2-E \cdot \mathrm{Dy}_{2}\right)$. In the syn isomer $f-\mathrm{P} 2-Z \cdot \mathrm{Dy}_{2}$, the steric clashes between the two adjacent Kläui ligands results in a tilting of the magnetic centers (Figure 3a). These steric interactions are absent in $f$ $\mathbf{P} 2-\boldsymbol{E} \cdot \mathrm{Dy}_{2}$, and the magnetic centers have an exactly anti-parallel alignment. The angles between the Co-Dy vector and the mean plane or the four porphyrin nitrogen atoms are $87.79(15)^{\circ}$ and $82.42(15)^{\circ}$ for the $s y n$ isomer, and $85.8(2)^{\circ}$ for the anti isomer. 

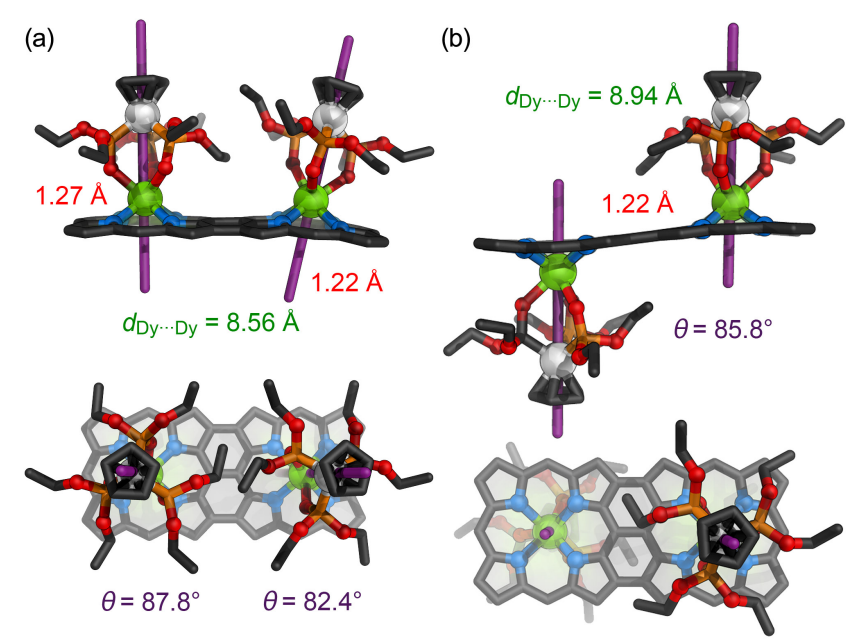

Figure 3. Crystal structure of (a) $f-\mathrm{P} 2-Z \cdot \mathrm{Dy}_{2}$ and (b) $\boldsymbol{f} \mathbf{P} 2-\boldsymbol{E} \cdot \mathrm{D} y_{2}$ from $\mathrm{X}$-ray diffraction studies showing side and top views. Solvent molecules, hydrogen atoms, aryl groups and minor components of disorder are omitted for clarity. The violet lines indicate the Co-Dy vectors. The distances of the Dy atoms from the mean planes of the four nitrogen atoms are shown in red and the angle $\theta$ of the Co-Dy vectors to the planes for the four nitrogen atoms are shown in purple.

Absorption Spectra. The absorption spectrum of $s-\mathbf{P} 2 \cdot \mathrm{Dy}_{2}$ is compared with that of its $\mathrm{Zn}^{\text {II }}$ analogue, $\boldsymbol{s}-\mathbf{P 2} \cdot \mathbf{Z n}_{\mathbf{2}}$ in Figure $4 \mathrm{a}$. The spectra are similar but that of $\boldsymbol{s}-\mathbf{P 2} \cdot \mathbf{D} \mathbf{y}_{2}$ is bathochromically shifted, with the lowest energy band maximum of $s-\mathbf{P} 2 \cdot \mathrm{Dy}_{2}$ at $625 \mathrm{~nm}$, versus $607 \mathrm{~nm}$ in $\boldsymbol{s}-\mathbf{P 2} \cdot \mathbf{Z n}_{2}$. This shift may be attributed to the smaller porphyrin-porphyrin torsion angle in the Dy ${ }^{\mathrm{III}}$ complex, as observed in the crystal structure, which allows more orbital overlap between the porphyrin $\pi$-systems. ${ }^{26,27}$
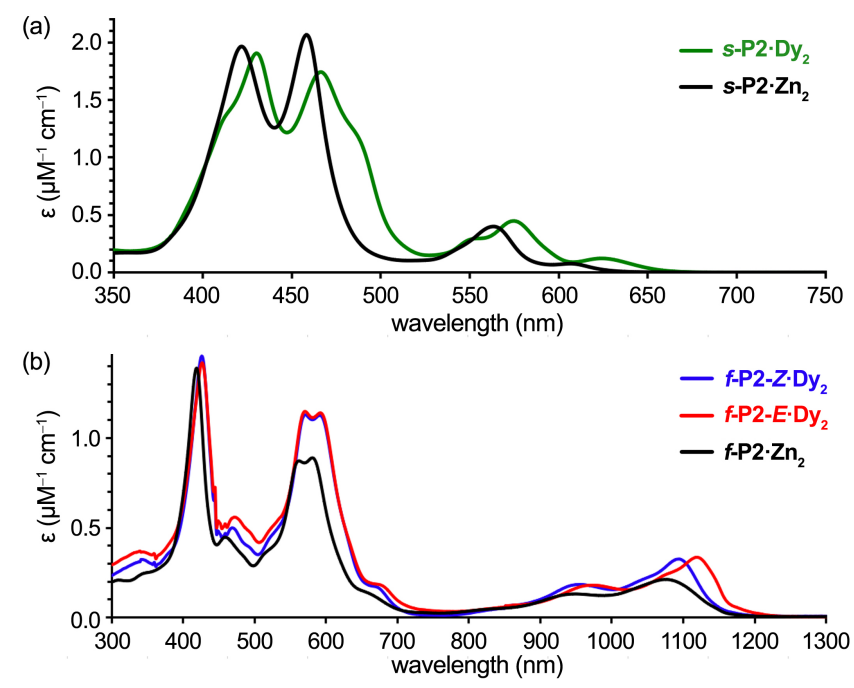

Figure 4. Absorption spectra of (a) the singly-linked dimers $s-\mathbf{P} 2 \cdot \mathbf{D} \mathbf{y}_{2}$ and $s-\mathbf{P 2} \cdot \mathrm{Zn}_{2}$ and (b) the edge-fused dimers $f \mathbf{P 2}-Z \cdot \mathrm{Dy}_{2}, f \mathbf{P 2}-\boldsymbol{E} \cdot \mathbf{D y _ { 2 }}$ and $f \mathbf{P} 2 \cdot \mathrm{Zn}_{2}$. Spectra recorded in $\mathrm{CHCl}_{3}$ at $20^{\circ} \mathrm{C}$.

Edge-fused porphyrin dimers generally exhibit $\pi-\pi^{*}$ absorption bands extending to wavelengths of $1100 \mathrm{~nm}$, reflecting strong $\pi$ conjugation. ${ }^{13}$ The absorption spectra of the syn and anti isomers $f$ $\mathrm{P} 2-Z \cdot \mathrm{Dy}_{2}$ and $f-\mathrm{P} 2-\boldsymbol{E} \cdot \mathrm{Dy}_{2}$ are similar to that of the corresponding $\mathrm{Zn}{ }^{\text {II }}$ complex (Figure $4 \mathrm{~b}$ ). The longest wavelength absorption band of the anti isomer, $f-\mathrm{P} 2-E \cdot \mathrm{Dy}_{2}\left(\lambda_{\max } 1121 \mathrm{~nm}\right)$, is red-shifted relative to the syn isomers, $f-P 2-Z \cdot D_{y_{2}}\left(\lambda_{\max } 1095 \mathrm{~nm}\right)$. In structurally related triply-linked corrole dimers bearing $\mathrm{Ga}^{\mathrm{III}}$, no difference in absorption behavior was observed between syn and anti isomers. ${ }^{28}$

Redox Potentials. The differential pulse voltammograms of $s$ $\mathrm{P} 2 \cdot \mathrm{Dy}_{2}, f-\mathrm{P} 2-Z \cdot \mathrm{Dy}_{2}$ and $f-\mathrm{P} 2-\boldsymbol{E} \cdot \mathrm{Dy}_{2}$ are compared with those of $s-$ $\mathbf{P 2} \cdot \mathbf{Z n}_{2}$ and $f \mathbf{P 2} \cdot \mathbf{Z n}_{2}$ in Figure 5. The first oxidation of $s-\mathbf{P 2} \cdot \mathbf{D y _ { 2 }}$ $\left(E_{\text {ox }}=0.12 \mathrm{~V}\right.$ vs. $\left.\mathrm{Fc} / \mathrm{Fc}^{+}\right)$is noticeably easier than that of $s-\mathbf{P 2} \cdot \mathbf{Z n}_{2}$ $\left(E_{\mathrm{ox}}=0.36 \mathrm{~V}\right)$, reflecting the fact that $\mathrm{Dy}^{\mathrm{III}}$ is more electropositive than $\mathrm{Zn}^{\text {II. }}{ }^{29}$ Both $\boldsymbol{s}$-P2 $\cdot \mathbf{D y}_{2}$ and $\boldsymbol{s}-\mathbf{P} 2 \cdot \mathbf{Z n}_{2}$ exhibits a total of five oxidation waves, and the potentials for the Dy ${ }^{\mathrm{III}}$ complex are more widely spaced, so that the $5^{\text {th }}$ oxidation potential of $s-\mathbf{P} 2 \cdot \mathbf{D y} y_{2}\left(E_{\text {ox }}=\right.$ $1.26 \mathrm{~V})$ is substantially higher than that of $\boldsymbol{s}-\mathbf{P} 2 \cdot \mathrm{Zn}_{2}\left(E_{\mathrm{ox}}=1.09 \mathrm{~V}\right)$. Almost identical behavior was observed for $\boldsymbol{s}-\mathbf{P 2} \cdot \mathbf{G d}_{2}$ (see Supporting Information, Figure S3).

The fused metalloporphyrin dimers $\mathbf{f} \mathbf{P} \mathbf{2} \cdot \mathbf{L n}_{2}$ and $\mathbf{f} \mathbf{P} \mathbf{2} \cdot \mathbf{Z n}_{\mathbf{2}}$ are easier to oxidize than the singly-linked dimers, reflecting their smaller HOMO-LUMO gaps. Four oxidation processes are observed in the window of accessible potentials. As for $\boldsymbol{s}-\mathbf{P} \mathbf{2} \cdot \mathbf{D y _ { 2 }}$, the lanthanide complexes are substantially easier to oxidize than $f$ $\mathbf{P 2} \cdot \mathbf{Z n}_{2}$. There is a significant shift in the first oxidation potential of $\boldsymbol{f}-\mathrm{P} 2-\boldsymbol{E} \cdot \mathrm{Dy}_{2}(-0.27 \mathrm{~V})$ relative to that of $\boldsymbol{f} \mathbf{P} 2-Z \cdot \mathrm{Dy}_{2}(-0.19 \mathrm{~V})$, indicating that the HOMO is higher in energy in the anti isomers. The other three oxidation potentials are almost the same for the syn and anti isomers. These data show the inclusion of $\mathrm{Ln}^{\mathrm{III}}$ metal centers does not disrupt the strong electronic coupling between adjoined porphyrins, and that the metal geometry fine-tunes the underlying electronic structure.
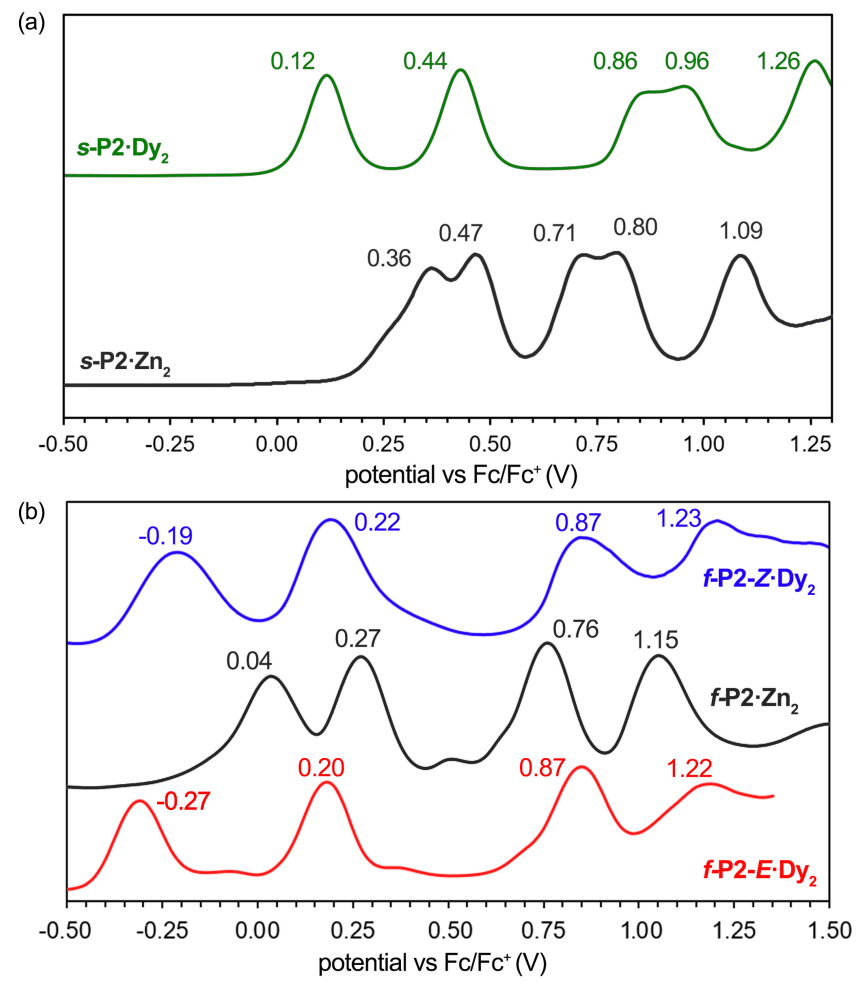

Figure 5. Differential pulse voltammograms of (a) $s-\mathbf{P 2} \cdot \mathbf{D} \mathbf{y}_{2}$ compared with $\boldsymbol{s}-\mathbf{P} 2 \cdot \mathbf{Z n}_{2}$, and (b) $\boldsymbol{f}-\mathbf{P} 2-Z \cdot \mathrm{Dy}_{2}$ and $\boldsymbol{f}-\mathbf{P} 2-\boldsymbol{E} \cdot \mathrm{Dy}_{2}$ compared with $\boldsymbol{f}$ $\mathbf{P 2} \cdot \mathrm{Zn}_{2}$. Solvent: $\mathrm{CH}_{2} \mathrm{Cl}_{2}$ containing $0.10 \mathrm{M} \mathrm{NBu}_{4} \mathrm{PF}_{6}$. 
The slightly higher HOMO and smaller optical HOMO-LUMO gap of the anti isomer could be attributed to the more regular molecular geometry of this isomer, due to the absence of any clash between the capped metal centers, as observed in the crystal structure.

Chiral Resolution and Circular Dichroism. Inserting $\mathrm{Ln}^{\mathrm{III}}$ into singly-linked porphyrin oligomers, with the capped $\mathrm{Ln}^{\mathrm{III}}$ metal center outside the porphyrin plane, generates two enantiomers. The chirality of these compounds opens up possibilities for unusual magnetic behavior such as magneto-chiral dichroism. ${ }^{30}$ While chiral singly-linked porphyrin oligomers are well-known, these structures are typically accessed through modification of pendant aryl groups or by preparing "strapped" porphyrins. ${ }^{26,31-33}$ To the best of our knowledge, this is the first example of metals acting as stereogenic elements in meso-linked porphyrin oligomers. We were pleased to find that enantiomers of $\boldsymbol{s}$-P2 $\cdot \mathbf{D y}_{2}$ can be resolved via chiral HPLC using a SUMICHIRAL OA-2500 stationary phase, which is functionalized with $(R)$-1-naphthylglycine (Figure 6a). With each enantiomer in hand, circular dichroism (CD) spectra were recorded (Figure 6b). These complexes possess three major Cotton effects of opposite signs. The most intense signal is located at 435 $\mathrm{nm}\left(\Delta \varepsilon \sim 600 \mathrm{M}^{-1} \mathrm{~cm}^{-1}\right)$, with two less intense signals at $400 \mathrm{~nm}$ $\left(\Delta \varepsilon \sim 110 \mathrm{M}^{-1} \mathrm{~cm}^{-1}\right)$ and $481 \mathrm{~nm}\left(\Delta \varepsilon \sim 70 \mathrm{M}^{-1} \mathrm{~cm}^{-1}\right)$. Very weak signals were also found at $575 \mathrm{~nm}\left(\Delta \varepsilon \sim 1 \mathrm{M}^{-1} \mathrm{~cm}^{-1}\right)$ and $625 \mathrm{~nm}$ $\left(\Delta \varepsilon \sim 5 \mathrm{M}^{-1} \mathrm{~cm}^{-1}\right.$ ) (Figure $6 \mathrm{c}$ ). Comparison of the $\mathrm{CD}$ spectra in Figure $6 \mathrm{~b}$ with that of a closely related meso-meso-linked zinc porphyrin dimer ${ }^{32}$ suggests that $(+)_{400-\boldsymbol{s}}-\mathbf{P} 2 \cdot \mathbf{D} \mathbf{D y}_{2}$ has the $S$ configuration. This very tentative assignment assumes that the electronic transition dipole moments of $\boldsymbol{s}-\mathbf{P 2} \cdot \mathbf{D y}_{2}$ are similar to those of the zinc porphyrin derivative. A more definitive assignment of the absolute configurations would require a time-dependent densityfunctional theory (TD-DFT) analysis, which is difficult with dysprosium complexes, or crystallographic analysis of resolved material.

We tested whether it is possible to thermally racemize these enantiomers. The chiral HPLC trace of a solution of $(+)_{400} \boldsymbol{s}-\mathbf{P} 2 \cdot \mathbf{D y}_{2}$ remained unchanged after heating to $200{ }^{\circ} \mathrm{C}$ for 24 hours in $\mathrm{O}_{2}$-free toluene in a sealed tube. There was no detectable racemization or decomposition, indicating that there is a high barrier to rotation about the central meso-meso single bond, as concluded previously for analogous zinc complexes. ${ }^{31}$ (a)
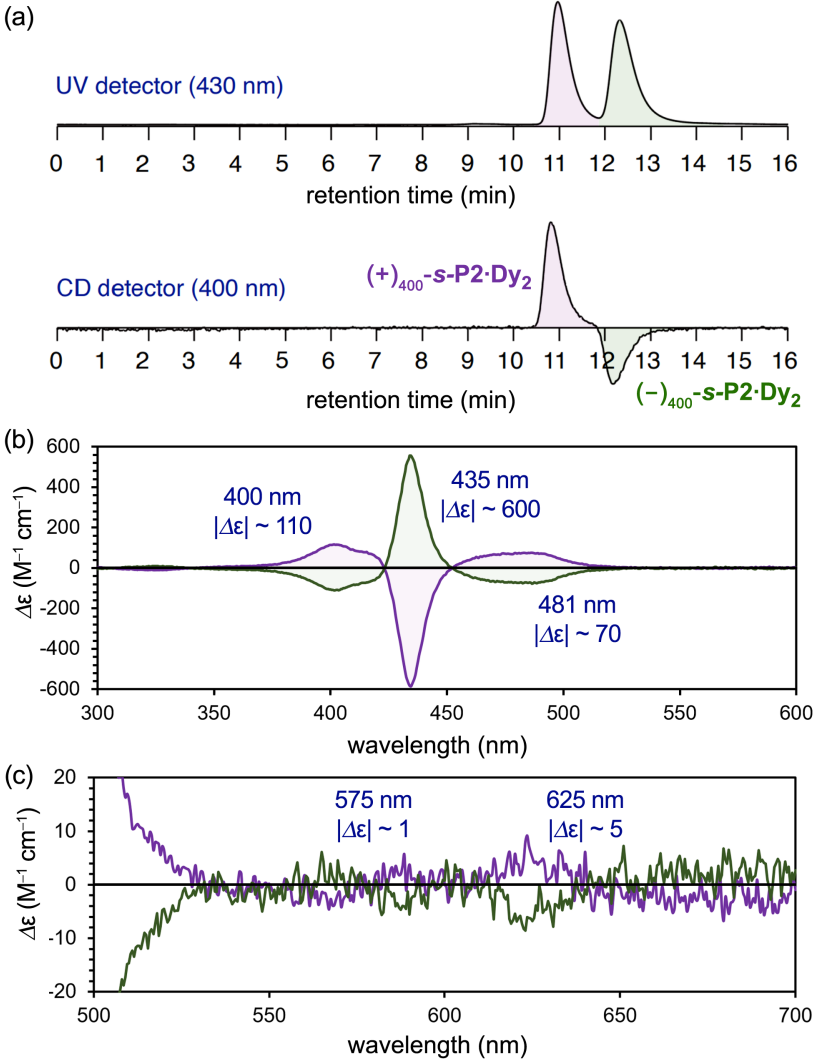

Figure 6. (a) Chiral HPLC trace of racemic $\boldsymbol{s}$-P2.Dy ${ }_{2}$ (stationary phase: SUMICHIRAL OA-2500; mobile phase: hexane/ $i-\operatorname{PrOH}(99: 1 \mathrm{v} / \mathrm{v})$; flow rate: $1.0 \mathrm{~mL} \mathrm{~min}^{-1}$; temperature $40{ }^{\circ} \mathrm{C}$; detector: $430 \mathrm{~nm}$ (top) and CD (bottom) (b) Full and (c) expanded CD spectra (concentration $4 \mu \mathrm{M}$; solvent $\mathrm{CHCl}_{3}$; temperature: $25^{\circ} \mathrm{C}$ ).

EPR Spectroscopy and Quantum Coherence. We evaluated the quantum coherence properties of the $\mathrm{Gd}^{\mathrm{III}}$ complexes by pulsed electron paramagnetic resonance (EPR) techniques, to explore whether the compounds could be suitable for quantum information processing at low temperatures. These experiments yield key parameters such as the spin-lattice relaxation time, $T_{1}$, and the spinspin dephasing (or phase-memory) time, $T_{\mathrm{m}}$. Pulsed EPR techniques can also provide valuable information on weak spin-spin dipolar and exchange interactions in dimeric systems. We restrict our analysis to the $\mathrm{Gd}^{\mathrm{III}}$ complexes, because of the extreme broadening and zero-field splitting of $\mathrm{Dy}^{\mathrm{III}}$ complexes. The orbital momentum is zero $(L=0)$ for ground state $\mathrm{Gd}^{\mathrm{III}}$ systems, so they can be treated as pure spin systems with a total spin of $S=7 / 2$. The electrostatic crystal-field environment splits the ground state into four Kramers doublets with $\left|m_{s}\right\rangle=| \pm 1 / 2\rangle,| \pm 3 / 2\rangle,| \pm 5 / 2\rangle$ and $| \pm 7 / 2\rangle$. Typically, for $\mathrm{Gd}^{\mathrm{III}}$ these splittings are relatively small $(\sim 100 \mathrm{GHz})$, so that all these states are populated at liquid helium temperatures. Furthermore, for $\mathrm{Gd}^{\mathrm{III}}$ complexes mixing between these states due to the crystal-field can be neglected and we can consider them as pure doublets. For these reasons, the quantum coherence properties are much more pronounced in $\mathrm{Gd}^{\mathrm{III}}$ ions, rather than in Dy ${ }^{\text {III }} \cdot{ }^{11,34}$ 
(a)

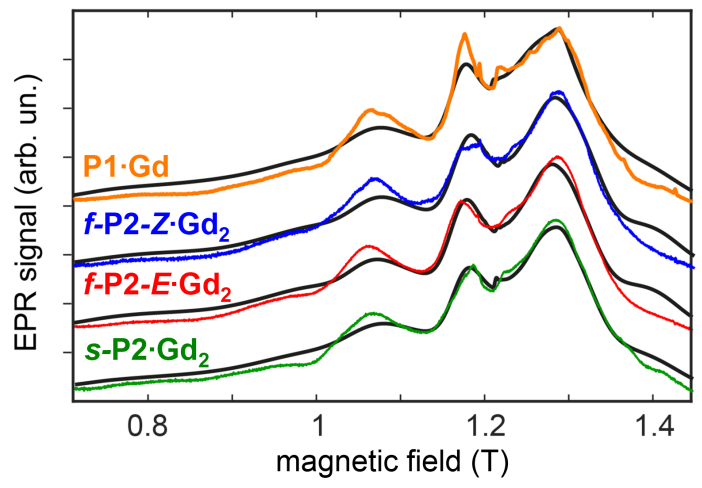

(b)

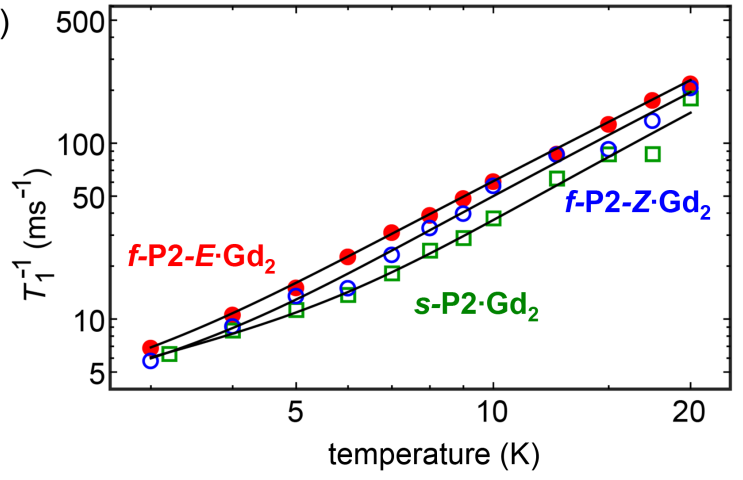

(c)

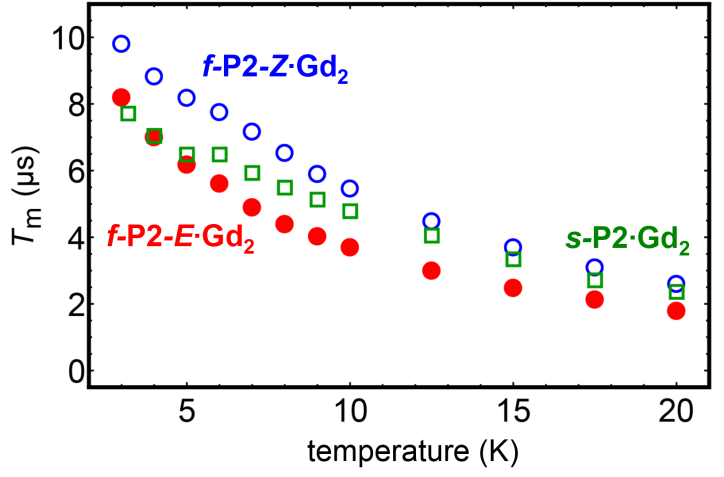

Figure 7. (a) Echo-detected field-swept Q-band EPR spectra for P1 $\cdot G d$ (orange), f-P2-Z $\cdot \mathbf{G d}_{2}$ (blue), f-P2-E$\cdot \mathbf{G d}_{2}$ (red) and $s \cdot \mathbf{P 2} \cdot \mathbf{G d}_{2}$ (green), acquired at $5 \mathrm{~K}$. Black lines are fits to the data based on the spin Hamiltonian (see SI). (b) Temperature-dependence of the spin-phonon relaxation rate $T_{1}^{-1}$ for $\boldsymbol{f} \mathbf{P} \mathbf{2}-\boldsymbol{E} \cdot \mathbf{G d}_{2}$, (red, full dots), $\boldsymbol{f} \mathbf{P} \mathbf{2}-\boldsymbol{Z} \cdot \mathbf{G d}_{2}$ (blue, open dots) and $s-\mathbf{P 2} \cdot \mathrm{Gd}_{2}$ (green, open rectangles), acquired at $B=$ $1.289 \mathrm{~T}, 1.287 \mathrm{~T}$ and $1.285 \mathrm{~T}$, respectively. Black lines are fits to the data to equation (1). (c) Temperature dependence of the phasememory time $T_{\mathrm{m}}$ for $\boldsymbol{f} \mathbf{- P 2}-\boldsymbol{E} \cdot \mathbf{G d}_{2}$, (red, full dots), $\boldsymbol{f} \mathbf{P} \mathbf{2}-\boldsymbol{Z} \cdot \mathbf{G d}_{2}$ (blue, open dots) and $s-\mathbf{P 2} \cdot \mathrm{Gd}_{2}$ (green, open rectangles), acquired at $B=$ $1.289 \mathrm{~T}, 1.287 \mathrm{~T}$ and $1.285 \mathrm{~T}$, respectively.

The EPR spectra of $\mathbf{P} 1 \cdot \mathrm{Gd}, f \cdot \mathbf{P} 2-Z \cdot \mathrm{Gd}_{2}, f \mathbf{P 2}-E \cdot \mathbf{G d}_{2}$ and $s$ $\mathbf{P 2} \cdot \mathrm{Gd}_{2}$ were recorded as $1 \mathrm{mM}$ solutions in $\mathrm{CS}_{2}$, at temperatures of 3-20 K, using an echo-detection technique. This solvent forms a glassy matrix below $160 \mathrm{~K}$. Spectra recorded at $5 \mathrm{~K}$ are shown in Figure 7a. Normally, $\mathrm{Gd}^{\mathrm{III}}$ complexes show a narrow, intense spectral feature originating from the $|-1 / 2\rangle \rightarrow|+1 / 2\rangle$ transition. $^{35}$ However, the Kläui ligands seem to induce an unusually strong crystal-field, and we found a strong zero-field splitting. Spectral calculations (see Figure S42) show that, for randomly oriented molecules as in a frozen solution, the different peaks cannot be clearly assigned to the magnetic states. For example, the $|-1 / 2\rangle \rightarrow$
$|+1 / 2\rangle$ transition varies between $1.16 \mathrm{~T}$ and $1.33 \mathrm{~T}$ due to its orientation dependence, giving rise to two large peaks. However, the field-orientation dependence of other allowed transitions is significantly stronger due to their larger magnetic state, thus overlapping with those transitions. However, the $|-1 / 2\rangle \rightarrow|+1 / 2\rangle$ is known to display a narrow linewidth when the molecular quantization axis is aligned with the magnetic field. This shows as a kink in the data around $1.22 \mathrm{~T}$ for the given frequency of $33.85 \mathrm{GHz}$. Differences observed in the spectra of $f \cdot \mathbf{P} 2-Z \cdot G_{2}, f-P 2-E \cdot G_{2}$ and $s-P 2 \cdot G_{2}$ can be explained by slight differences of the crystal-field parameters. All the spectra could be fitted using a model that accounts for the crystal-field splitting parameters $D$ and $E$, and associated strains, as well as an isotropic $g$-factor and exchange coupling $J$ mediated by the bridging ligands (see SI, Table S7). The crystalfield splitting and the strong orientation dependence dominate the shapes of the spectra, and, consequently, the coupling strengths deduced from fitting these spectra have large error bars. $D$ and $E$ are similar for all three Gd complexes, with values between -3518 $\mathrm{MHz}$ and $-3590 \mathrm{MHz}$ for $D$, and between $249 \mathrm{MHz}$ and $304 \mathrm{MHz}$ for $E$ (see Table S7). In the fused dimers, the exchange coupling between the spins is very weak and antiferromagnetic. Fitting the spectra gives $J=-21$ and $J=-24 \mathrm{MHz}$ for $f \mathbf{P 2}-Z \cdot \mathbf{G d}_{2}$ and $f \mathbf{P} 2$ $E \cdot G_{2}$, respectively, but these values must be regarded as very approximate. More accurate values of the exchange coupling in these compounds were estimated from the low-temperature static magnetic susceptibility data, as discussed below. For the singlylinked dimer $\boldsymbol{s}-\mathbf{P 2} \cdot \mathbf{G d}_{2}$, exchange coupling was not detected, as expected, owing to the negligible orbital overlap of the two porphyrin conjugated systems.

The spin-lattice relaxation rate, $1 / T_{1}$ shows a rather linear increase with temperature below $6 \mathrm{~K}$ (Figure $7 \mathrm{~b}$ ). At temperatures above $6 \mathrm{~K}, 1 / T_{1}$ follows a polynomial law $T^{n}$ with $n>>1$, typical for Raman processes. Fitting with a combination of a direct and a Raman relaxation process could be performed, ${ }^{36}$ showing good agreement with equation (1) from $3 \mathrm{~K}$ to $20 \mathrm{~K}$ (see SI).

$$
T_{1}^{-1}=a_{0} T+a_{1}\left(\frac{T}{\theta_{D}}\right)^{9} \int_{0}^{\theta_{D} / T} \frac{x^{8} \mathrm{e}^{x}}{\left(\mathrm{e}^{x}-1\right)^{2}} \mathrm{~d} x
$$

Here, $a_{0}$ corresponds to the direct relaxation rate constant, $a_{1}$ to the Raman relaxation rate constant, $\theta_{\mathrm{D}}$ the Debye temperature and $T$ the temperature. We found $a_{0}=1.90(27) \mathrm{K}^{-1} \mathrm{~s}^{-1}, a_{1}=1503(834)$ $\mathrm{s}^{-1}, \theta_{\mathrm{D}}=22.5(55) \mathrm{K}$ for $\boldsymbol{f}$-P2-Z.G. $\mathrm{Gd}_{2} ; a_{0}=2.07(19) \mathrm{s}^{-1}, a_{1}=$ $1118(353) \mathrm{s}^{-1}, \theta_{\mathrm{D}}=18.0(27) \mathrm{K}$ for $\boldsymbol{f}$-P2-E.Gd $\mathrm{Gd}_{2}$ and $a_{0}=2.01(17) \mathrm{s}^{-}$ ${ }^{1}, a_{1}=2627(1423) \mathrm{s}^{-1}, \theta_{\mathrm{D}}=33.9(71) \mathrm{K}$ for $\boldsymbol{s}$-P2.Gd ${ }_{2}$. Below $6 \mathrm{~K}$, a slight deviation is observed, particularly for $\boldsymbol{f} \mathbf{- P 2}-\boldsymbol{E} \cdot \mathrm{Gd}_{2}$, as is typical for direct processes, in agreement with the processes limiting the ac spin dynamics of the $\mathrm{Dy}^{\text {III }}$ analogues (see below).

The phase-memory times, $T_{\mathrm{m}}$, were measured using a Hahn-echo sequence (see SI). Fitting the signal decay was performed with a mono-exponential decay function. The $T_{\mathrm{m}}$ times increase sharply on lowering $T$, until, at $3 \mathrm{~K}$, they reach up to $9.8 \mu$ for $\boldsymbol{f} \mathbf{P} \mathbf{P}-Z \cdot \mathbf{G d}_{2}$ and $8.2 \mu \mathrm{f} f \mathbf{P} 2-E^{\cdot} \mathbf{G d}_{2}$ (Figure $7 \mathrm{c}$ ), which is sufficiently long to test quantum computational schemes using microwave pulses. The $T_{\mathrm{m}}$ times for $\boldsymbol{s} \mathbf{s} \mathbf{2} \cdot \mathrm{Gd}_{2}$ lie in between those of the fused dimers. $T_{\mathrm{m}}$ slightly depends on the applied magnetic field $B$ because different states and transitions can be selected and probed (see SI). In the whole region, we are still far below the coherence time limit (i.e. $T_{2}$ $<<2 T_{1}$, where $T_{2}$ is the quantum coherence time, a major contributing factor to $T_{\mathrm{m}}$ ), indicating that hyperfine interactions with ${ }^{155,157} \mathrm{Gd},{ }^{14,15} \mathrm{~N}$ and ${ }^{31} \mathrm{P}$ probably dominate the decoherence process. 
Even in this limit, the complex would allow ca. 250 two-quantumbits operations to be performed within the time constrictions imposed by the spin-spin interactions. Interestingly, although the spin-spin interactions of the syn and anti complexes are very similar, the different symmetries introduced by the two configurations have an effect on the quantum coherence. In the whole temperature range examined, $\boldsymbol{f} \mathbf{- P 2}-\boldsymbol{Z} \cdot \mathbf{G d}_{2}$ always displays substantially longer coherence than $\boldsymbol{f} \mathbf{P} \mathbf{2}-\boldsymbol{E} \cdot \mathrm{Gd}_{2}$ (up to $30 \%$ longer). Intermolecular interactions can be ruled out since the molecules are spaced far enough apart in a $1 \mathrm{mM}$ frozen glassy solution. The effect is noteworthy, as several proposals rely on slightly-tilted neighboring spins for two-qubit operations. ${ }^{37}$

Static Magnetic Properties. The variable temperature magnetic properties of all the complexes were determined using a MPMS-XL SQUID magnetometer. The dependence of the static magnetic susceptibility, $\chi_{\mathrm{M}}$, on temperature $T$, is shown in Figure 8 for all compounds: $\mathrm{P} 1 \cdot \mathrm{Dy}, s-\mathrm{P} 2 \cdot \mathrm{Dy}_{2}, f-\mathrm{P} 2-Z \cdot \mathrm{Dy}_{2}, f-\mathrm{P} 2-E \cdot \mathrm{Dy}_{2}, \mathrm{P} 1 \cdot \mathrm{Gd}, s$ $\mathrm{P} 2 \cdot \mathrm{Gd}_{2}, f-\mathrm{P} 2-Z \cdot \mathrm{Gd}_{2}$, and $f-\mathrm{P} 2-E \cdot \mathrm{Gd}_{2}$, where, $\chi_{\mathrm{M}}$ is the ratio between the magnetization $M$ and the applied external magnetic field $B$. Dy ${ }^{\text {III }}$ ions have a ${ }^{6} \mathrm{H}_{15 / 2}$ ground state configuration, and very large spin-orbit coupling leads to the presence of several Kramers doublets that are split by anisotropy, while $\mathrm{Gd}^{\mathrm{III}}$ ions are in the ${ }^{8} \mathrm{~S}_{0}$ configuration and thus lack any spin-orbit contribution. The $\chi_{M} T$ values at $300 \mathrm{~K}$ for the monomers (Table 1) agree with the expected values for a single $\mathrm{Dy}^{\mathrm{III}}$ or $\mathrm{Gd}^{\mathrm{III}}$ ion (14.2 and $7.9 \mathrm{emu} \mathrm{K}$ $\mathrm{mol}^{-1}$, respectively), ${ }^{38}$ and the values for the dimers are close to twice those of the monomers, as expected for two non-interacting ions. In all the Dy compounds $\chi_{\mathrm{M}} T$ decreases slightly on cooling, with a steep decrease below $100 \mathrm{~K}$ (Figure 8a). This decrease is mainly linked to the depopulation of the excited Stark sublevels of the $\mathrm{Dy}^{\mathrm{III}}$, as revealed by comparing the curves of the dimers to twice that of the monomer. ${ }^{39}$ For $\mathbf{P 1} \cdot \mathrm{Gd}, \chi_{\mathrm{M}} T$ remains constant from 300 $\mathrm{K}$ to $16 \mathrm{~K}$ and then decreases slightly to $7.7(2) \mathrm{emu} \mathrm{K} \mathrm{mol}^{-1}$ (Figure $8 b)$. For the $\mathrm{Gd}_{2}$ dimers, $\chi_{\mathrm{M}} T$ also remains almost constant until 16 $\mathrm{K}$, below which temperature it decreases to reach a value of $15.6(4)$ emu K mol ${ }^{-1}$ for $s-\mathbf{P 2} \cdot \mathrm{Gd}_{2}, 15.2(5)$ emu $\mathrm{K} \mathrm{mol}^{-1}$ for $\boldsymbol{f} \mathbf{P} 2-Z \cdot \mathbf{G d}_{2}$ and $15.5(5) \mathrm{emu} \mathrm{K} \mathrm{mol}{ }^{-1}$ for $\boldsymbol{f}-\mathrm{P} 2-\boldsymbol{E} \cdot \mathrm{Gd}_{2}$ at $T=2 \mathrm{~K}$. This low- $T$ decrease suggests the presence of weak intramolecular antiferromagnetic interactions. As the monomer curves always lie between the curves of the syn and anti isomers, it is tempting to attribute ferromagnetic interactions to $\boldsymbol{f} \mathbf{P} \mathbf{2}-\boldsymbol{Z} \cdot \mathrm{Dy}_{2}$, and antiferromagnetic ones to $f-\mathrm{P} 2-\boldsymbol{E} \cdot \mathrm{Dy}_{2}$. On the other hand, the EPR analysis indicates that the interactions are in the range -21 to $-24 \mathrm{MHz}$, and the effect should rather be attributed to changes in the anisotropy, as produced by the considerable distortion of the coordination environment in $f$ $\mathrm{P} 2-Z \cdot \mathrm{Dy}_{2}$.

Magnetization curves were recorded for all complexes at 2, 5 and $7 \mathrm{~K}$ up to $7 \mathrm{~T}$ (Figures S7-S14). The $M$ versus $B$ curves show a rapid increase at low fields (below $1 \mathrm{~T}$ ) for all $\mathrm{Dy}^{\mathrm{III}}$ complexes, followed by a slow, almost linear increase at high fields. For $\mathrm{Dy}^{\mathrm{III}}$ complexes, the $M$ vs. $B$ curves agree well with the simulations that include the presence of magnetic anisotropy. In the case of the isotropic complexes $s-\mathrm{P2} \cdot \mathrm{Gd}_{2}, f-\mathrm{P} 2-Z \cdot \mathrm{Gd}_{2}$, and $f-\mathrm{P} 2-E \cdot \mathrm{Gd}_{2}$ (Figures $\mathrm{S} 12-\mathrm{S} 14)$, even at $2 \mathrm{~K}$ the magnetization shows a rapid increase and reaches a saturation value expected for non-interacting $\mathrm{Gd}^{\mathrm{III}}$ ions, in agreement with the very weak interactions detected by $\mathrm{EPR}^{36}$
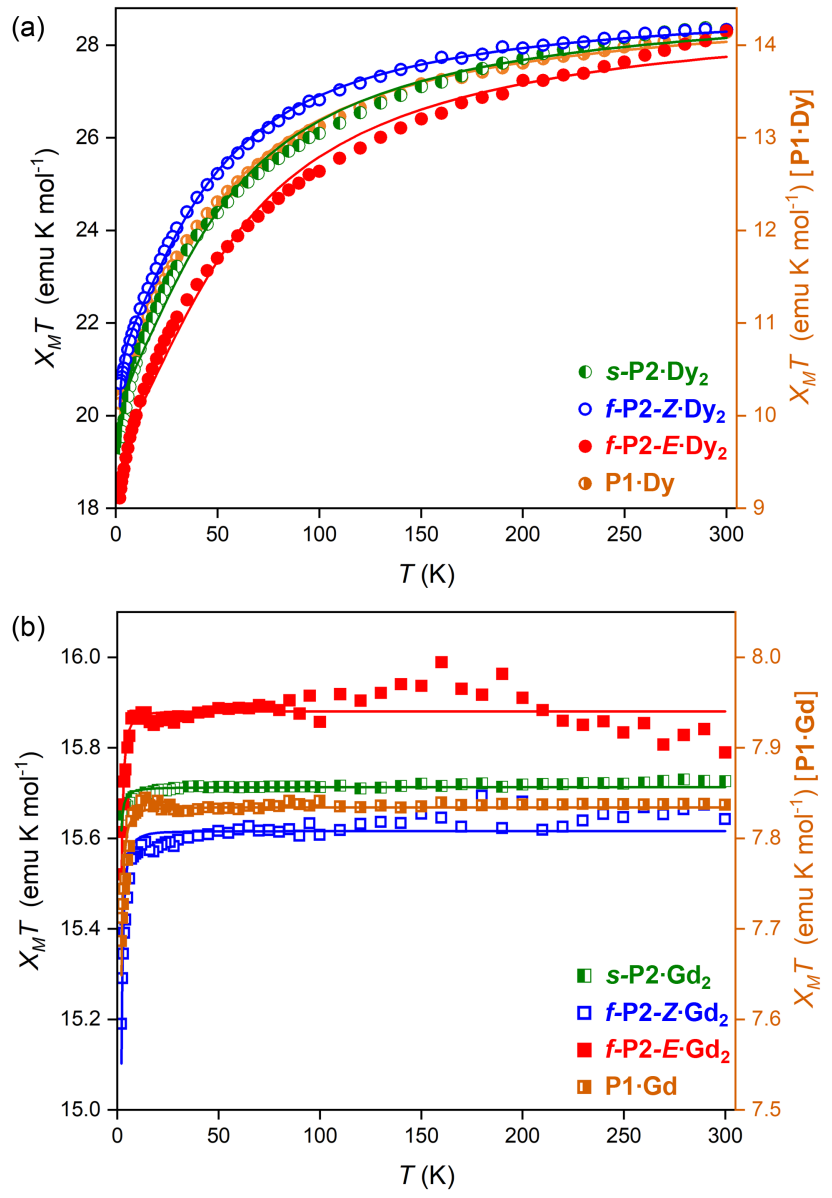

Figure 8. Temperature dependence of the static magnetic susceptibility for complexes $s-\mathrm{P} 2 \cdot \mathrm{Dy}_{2}, f-\mathrm{P} 2-Z \cdot \mathrm{Dy}_{2}, f-\mathrm{P} 2-E \cdot \mathrm{Dy}_{2}, s-\mathrm{P} 2 \cdot \mathrm{Gd}_{2}, f-\mathrm{P} 2-Z \cdot \mathrm{Gd}_{2}$ and $\boldsymbol{f}-\mathrm{P} 2-\boldsymbol{E} \cdot \mathrm{Gd}_{2}$ and for their respective monomers $\mathrm{P} \mathbf{1} \cdot \mathrm{Dy}$ and $\mathrm{P} \mathbf{1} \cdot \mathrm{Gd}$. All curves acquired in a static field $B=0.1 \mathrm{~T}$. Solid lines are fits.

Table 1. Static magnetic susceptibilities, measured at $300 \mathrm{~K}$.

\begin{tabular}{|c|c|}
\hline complex & $\chi_{\mathrm{M}} T / \mathrm{emu} \mathrm{K} \mathrm{\textrm {mol } ^ { - 1 }}$ \\
\hline P1.Dy & $14.1(4)$ \\
\hline$s-\mathrm{P} 2 \cdot \mathrm{Dy}_{2}$ & $28.3(4)$ \\
\hline$f-\mathrm{P} 2-Z \mathrm{D} \mathrm{y}_{2}$ & $28.3(8)$ \\
\hline$f-\mathrm{P} 2-E \cdot D y_{2}$ & $28.3(8)$ \\
\hline P1.Gd & $7.8(2)$ \\
\hline$s$-P2.Gd ${ }_{2}$ & $15.7(4)$ \\
\hline$f-\mathrm{P} 2-Z \cdot \mathrm{Gd}_{2}$ & $15.8(5)$ \\
\hline$f-\mathrm{P} 2-E \cdot \mathrm{Gd}_{2}$ & $15.6(5)$ \\
\hline
\end{tabular}

For $\mathrm{P} 1 \cdot \mathrm{Gd}, s-\mathrm{P} 2 \cdot \mathrm{Gd}_{2}, f-\mathrm{P} 2-Z \cdot \mathrm{Gd}_{2}$, and $f-\mathrm{P} 2-E \cdot \mathrm{Gd}_{2}$, the magnetic data were fitted using a model that accounts for the isotropic $g$ factor, an isotropic exchange coupling parameter $J$ for the dimers, and the zero-field splitting parameters $D$ and $E$ as determined from EPR (Table S2). The inclusion of $J$ improves the fit quality (Figure $8 \mathrm{~b})$. We found values of $g=1.9942(55)$ for $\mathrm{P1} \cdot \mathrm{Gd}, g=$ $1.9980(201)$ and $J=0 \mathrm{MHz}$ for $\boldsymbol{s}-\mathbf{P 2} \cdot \mathbf{G d}_{2}, g=1.9919(127)$ and $J=$ $(-51 \pm 2) \mathrm{MHz}$ for $\boldsymbol{f} \mathbf{P} \mathbf{2}-\boldsymbol{Z} \cdot \mathbf{G d}_{2}$ and $g=2.0082(382)$ and $J=(-19 \pm$ 3) $\mathrm{MHz}$ for $f-\mathrm{P} 2-\boldsymbol{E} \cdot \mathrm{Gd}_{2}$. The $J$ values of the fused dimers come close to the EPR results and indicate a small antiferromagnetic exchange. These interactions fall in the useful range for twoquantum-bit operations, which for the values above could be per- 
formed at $40 \mathrm{~ns}$. These values are thus encouraging for the perspective use of porphyrin scaffolds, and would allow 30 times the operations of previously-proposed bimetallic complexes. ${ }^{40}$ Best fits for the singly-linked dimer are obtained when neglecting exchange interactions. Data of the Dy analogues were fitted using a simplified ligand-field model considering only second-order zero-fieldsplitting parameters, but over-parametrization limits the reliability in determining the exchange. We found good agreement using $S=$ $15 / 2$ with $g=1.3449(63), D=(-510.0 \pm 0.4) \mathrm{GHz}$ and $E=(603.9$ $\pm 0.5) \mathrm{MHz}$ for P1 $\cdot \mathrm{Dy} ; g=1.3484(96), D=(-558.0 \pm 46.2) \mathrm{GHz}$ and $E=(645.2 \pm 51.7) \mathrm{GHz}$ for $s$-P2 $\cdot \mathrm{Dy}_{2} ; g=1.3457(233), D=(-$ $531.2 \pm 156.2) \mathrm{GHz}$ and $E=(449.5 \pm 30.7) \mathrm{GHz}$ for $\boldsymbol{f} \mathbf{P} 2-\boldsymbol{Z} \cdot \mathbf{D y}_{2} ; g$ $=1.3445(83), D=(-870.1 \pm 145.4) \mathrm{GHz}$ and $E=(779.7 \pm 36.7)$ $\mathrm{GHz}$ for $\boldsymbol{f}-\mathrm{P} 2-\boldsymbol{E} \cdot \mathrm{Dy}$.

Dynamic Susceptibilities. Alternating current (ac) magnetic susceptibility measurements were performed to probe the dynamics of the anisotropic compounds, P1.Dy, $s-\mathrm{P} 2 \cdot \mathrm{Dy} y_{2}, f-\mathrm{P} 2-Z \cdot \mathrm{Dy} y_{2}$ and $f-\mathrm{P} 2-$ $E \cdot \mathrm{Dy}_{2}$, and to check for slow relaxation of magnetization. We used a $0.2 \mathrm{mT}$ oscillating field at variable frequencies $v=1-1000 \mathrm{~Hz}$. None of the complexes exhibit in-phase $\left(\chi^{\prime}\right)$ or out-of-phase $\left(\chi^{\prime \prime}\right)$ susceptibility signals at zero static field, between $2-20 \mathrm{~K}$, (Figures S15-S17). In the Dy ${ }^{\text {III }}$ complexes this is typical of significant quantum tunneling of the magnetization (QTM). In order to suppress this QTM behavior, ac susceptibility measurements were performed as a function of $B$, showing a well-resolved maximum in $\chi^{\prime \prime}$ at $B=0.12 \mathrm{~T}$ (Figures S19-S34). At this field, all Dy complexes show the presence of peaks that shift to lower $v$ on decreasing $T$, as indicative of slow relaxation of the magnetization, or singlemolecule-magnet behavior, produced by the presence of a magnetic anisotropy barrier that hinders the reversal of the spin at the singlemolecule level (Figure 9).

The Argand plots display semicircular shapes that fit to a generalized Debye model for $\mathrm{P} 1 \cdot \mathrm{Dy}, \boldsymbol{s}-\mathrm{P} 2 \cdot \mathrm{D} \mathrm{y}_{2}, \boldsymbol{f}-\mathrm{P} 2-Z \cdot \mathrm{Dy}_{2}$ and $f-\mathrm{P} 2-E \cdot \mathrm{Dy}_{2}$ (Figures S25, S28, S31 and S34). ${ }^{41}$ The model includes an $\alpha$ parameter indicating a distribution of relaxation times, and is always in the range $0.1-0.3$ for all the Dy complexes (Tables S3-S6), implying a single relaxation process. The Arrhenius plots could be fitted with an Arrhenius law modified to include quantum tunneling rate $\tau_{Q T}^{-1}$, a Raman process $C T^{n}$, in addition to the Orbach relaxation channel:

$$
\tau^{-1}=\tau_{Q T}^{-1}+C T^{n}+\tau_{0}^{-1} e^{\frac{U_{e f f}}{k_{B} T}}
$$

where $U_{\text {eff }}$ is the relaxation energy barrier, $\tau_{0}$ is a pre-exponential factor, $k_{\mathrm{B}}$ is the Boltzmann constant, $C$ is a parameter containing the spin-phonon coupling matrix element, $n$ is the standard Raman exponent and is expected to be 9 for Kramers ions, or 5 in the presence of low-lying states. ${ }^{42}$ Best-fit parameters are shown in Table 2.

Table 2. Fitting parameters for compounds $\mathrm{P} 1 \cdot \mathrm{Dy}, \boldsymbol{s}-\mathrm{P} 2 \mathrm{Dy} \mathbf{y}_{2}, \mathrm{f}-\mathrm{P2}$ $Z \cdot \mathrm{Dy}_{2}$, and $f \cdot \mathrm{P} 2-E \cdot \mathrm{Dy}_{2}$ relating to equation (2).

\begin{tabular}{lllll}
\hline & P1.Dy & $s$-P2.Dy & $f-P 2-Z \cdot D y_{2}$ & $f-P 2-E \cdot$ Dy $_{2}$ \\
\hline$\tau_{\mathrm{QT}} / \mathrm{s}$ & $3.9(33) \times 10^{-3}$ & $3.5(5) \times 10^{-4}$ & $3.4(7) \times 10^{-3}$ & $1.1(5) \times 10^{-3}$ \\
$n$ & 9 & 9 & 9 & 9 \\
$C / \mathrm{s}^{-1} \mathrm{~K}^{-\mathrm{n}}$ & $9.7(5) \times 10^{-3}$ & $2.23(24) \times 10^{-2}$ & $2.5(15) \times 10^{-3}$ & $8.9(7) \times 10^{-3}$ \\
$\tau_{0} / \mathrm{s}$ & $1.7(5) \times 10^{-6}$ & $1.44(1) \times 10^{-5}$ & $3.0(3) \times 10^{-6}$ & $6.6(34) \times 10^{-6}$ \\
$U_{\mathrm{eff}} / \mathrm{K}$ & $9.8(12)$ & $10.4(10)$ & $8.9(4)$ & $10.1(17)$ \\
\hline
\end{tabular}
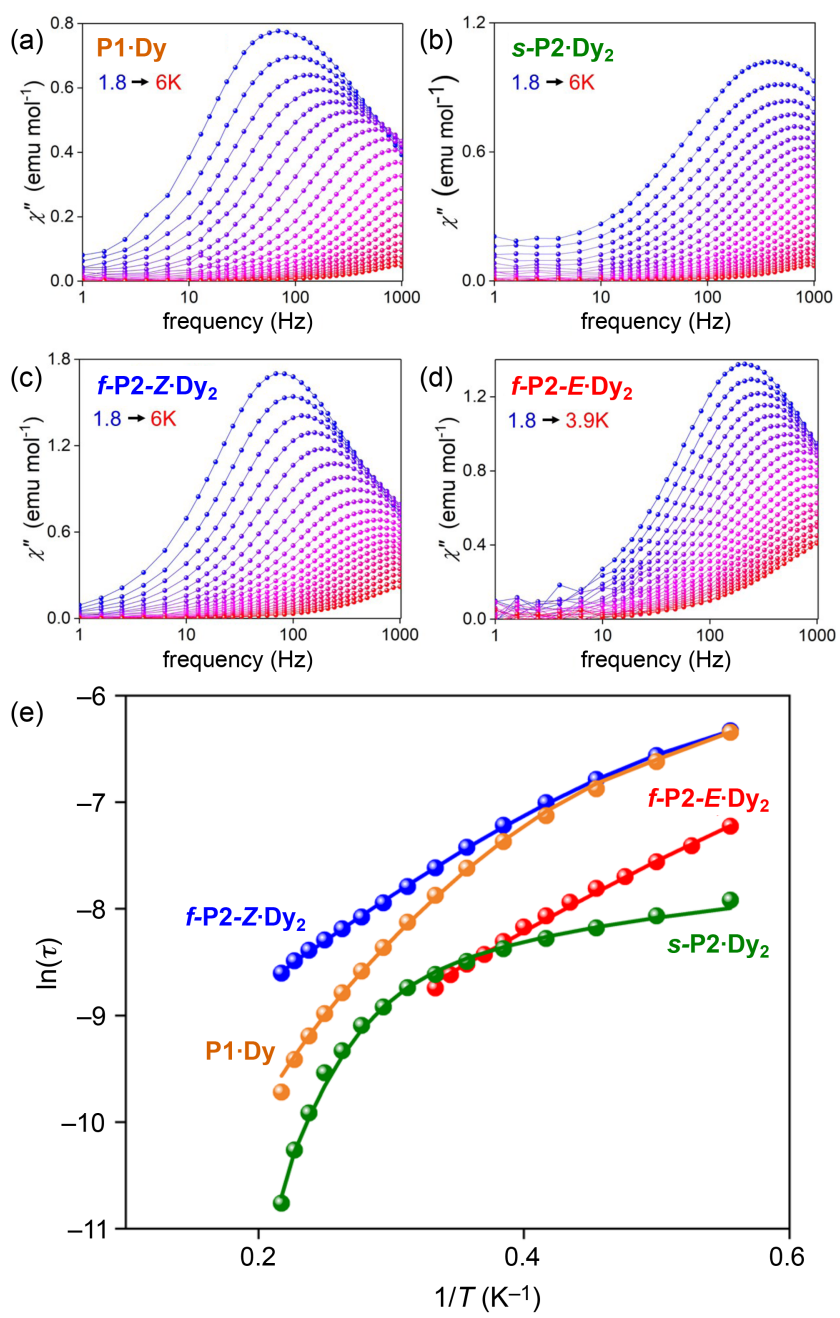

Figure 9. Frequency dependence of the out-of-phase signal of (a) $\mathrm{P} 1 \cdot \mathrm{Dy}$, (b) $s-\mathrm{P} 2 \cdot \mathrm{Dy}_{2}$, (c) $f-\mathrm{P} 2-Z \cdot \mathrm{Dy}_{2}$, and (d) $f-\mathrm{P} 2-\boldsymbol{E} \cdot \mathrm{D} \mathbf{y}_{2}$, solid lines are guides for the eye. (e) Comparison of the Arrhenius plots of P1 $\cdot D y$, $s$-P2 $\cdot \mathrm{Dy}_{2}, f-\mathrm{P} 2-Z \cdot \mathrm{Dy}_{2}$ and $f-\mathrm{P} 2-E \cdot \mathrm{Dy}_{2}$. Solid lines highlight fits to a modified Arrhenius equation including tunneling (see text).

This analysis reveals that bimetallic complexes $s-\mathrm{P2} \cdot \mathrm{Dy} 2, \mathrm{f}-\mathrm{P2}$ $Z \cdot \mathrm{Dy}_{2}$ and $\boldsymbol{f}-\mathrm{P} 2-\boldsymbol{E} \cdot \mathrm{Dy}_{2}$ show similar activation dynamics. The relaxation barriers for $\boldsymbol{f}-\mathrm{P} \mathbf{2}-\boldsymbol{Z} \cdot \mathrm{Dy}_{\mathbf{2}}$ and $\boldsymbol{f}-\mathrm{P} \mathbf{2}-\boldsymbol{E} \cdot \mathbf{D} \mathbf{y}_{2}$ are comparable with the monometallic complex P1 Dy, slightly higher than those of the butadiyne-linked $\mathrm{Dy}_{2}$ porphyrin dimers, ${ }^{11}$ and similar to that reported for the 10,15,20-tetraphenylporphyrin dysprosium complex. $^{20}$ The $\tau_{\mathrm{QT}}$ values suggest that quantum tunneling of the magnetization dominates in the low-temperature regime for all the complexes, although it is reduced to some extent by the application of $B$. For all complexes, the fitted model agrees excellently with the prediction for Kramers ions. ${ }^{43}$

Low Temperature Magnetic Anisotropy. The largest effects of the symmetry changes introduced by the aromatic plane are likely to arise in the magnetic anisotropy. The in-built magnetic anisotropy of the molecule will lead to a preferential orientation of the magnetization along the anisotropy axis, and will thus give rise to a magnetic torque, $\zeta=M \times B$, which will tend to move the crystal to align $M$ along $B$. A complete characterization of the torque response as a function of the orientation of $B$ and $T$ was thus performed on both $\boldsymbol{f} \mathbf{P} \mathbf{2}-\boldsymbol{Z} \cdot \mathrm{Dy}_{2}$ and $\boldsymbol{f}-\mathrm{P} \mathbf{2}-\boldsymbol{E} \cdot \mathrm{Dy}_{2}$ at milli-kelvin temperatures, as shown in Figure 10. The torque signal is measured via the 
deflection of a $50 \mu \mathrm{m}$-thick $\mathrm{CuBe}$ cantilever, induced by a magnetic field $B$, and measured as the variation of the capacitance with an underlying conductive plate (SI). Single crystals of both isomers were measured in two different crystal orientations each: in a plane approximately perpendicular and parallel to the porphyrin plane (Figure 10 ). Whatever the orientation, $f-P 2-E \cdot D y_{2}$ displays the torque behavior characteristic to a paramagnet with $180^{\circ}$ periodicity and approximately the same magnitude of positive and negative torque at extremal points. $\mathbf{f} \mathbf{P} \mathbf{2}-\boldsymbol{Z} \cdot \mathbf{D} \mathbf{y}_{2}$ displays a torque signal that is almost always positive, and is distinguished by a region of almost $180^{\circ}$ without any torque inversion. (a)

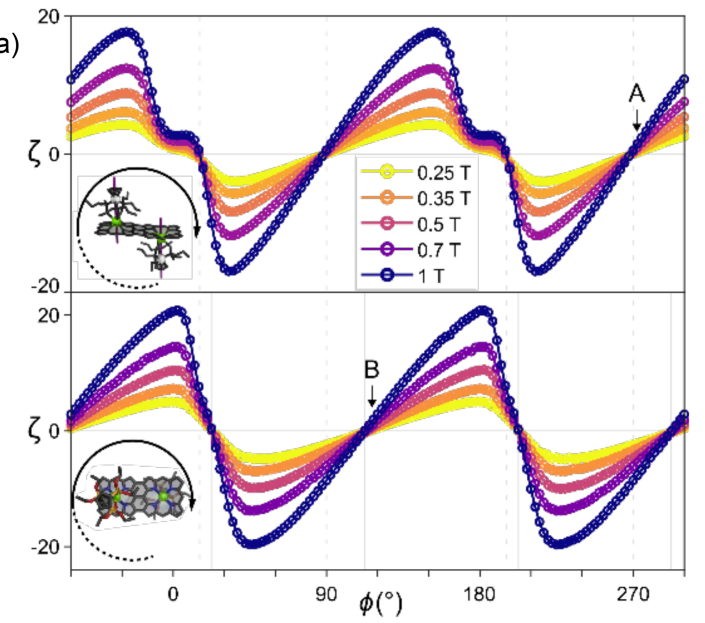

(b)

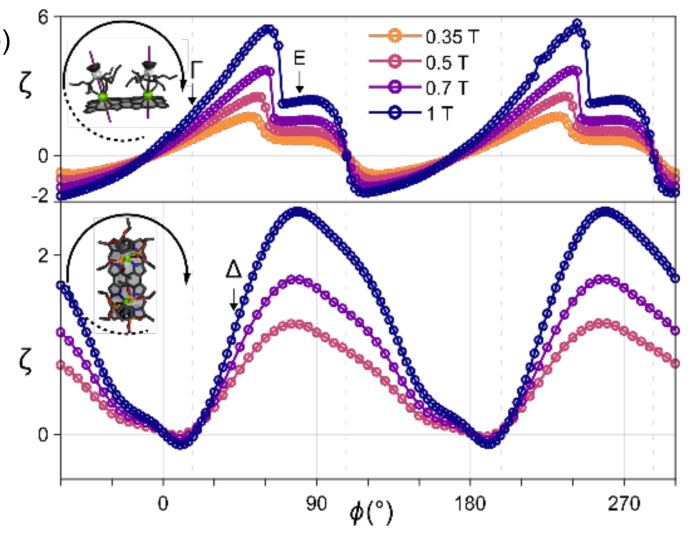

Figure 10. (a) Angular dependence of the magnetic cantilever-torque signal $\zeta$ of $\boldsymbol{f}-\mathrm{P} 2-\boldsymbol{E} \cdot \mathrm{Dy}_{2}$, measured at $50 \mathrm{mK}$ at different fields (color scale common to both panels). The two panels depict two rotations acquired for two different orientations of the crystal. The directions of the rotation with respect to the molecular orientation are depicted in the insets. Symbols denote the main angle for which the hysteresis loops are shown in Figure 11. (b) Angular dependence of the magnetic torque signal $\zeta$ of $\mathbf{f} \mathbf{P} \mathbf{2}-\boldsymbol{Z} \cdot \mathrm{D} \mathrm{Dy}_{2}$, measured at $50 \mathrm{mK}$ at different fields (color scale common to both panels). The two panels depict two rotations acquired for two different orientations of the crystal. The direction of the rotation with respect to the molecular orientation are depicted in the insets. Symbols denote the main angle for which the hysteresis loops are shown in Figure 11.
Both $f-\mathrm{P} 2-Z \cdot \mathrm{Dy}_{2}$ and $\boldsymbol{f} \mathrm{P} 2-\boldsymbol{E} \cdot \mathrm{Dy_{2 }}$ crystallize with two molecules per unit cell $(Z=2)$, but a crystallographic inversion center is present, so that the two molecules are equivalent. Moreover, the intramolecular inversion center in $f-\mathrm{P} 2-E \cdot D y_{2}$ makes the two $\mathrm{Dy}^{\mathrm{III}}$ magnetic centers equivalent, and their anisotropy axes must be collinear. Therefore, $f \mathbf{P} 2-E \cdot D y_{2}$ contains only one type of center, with all the anisotropy axes exactly aligned. For the purposes of torque magnetometry, the crystal response is thus equivalent to a single Dy ${ }^{\text {III }}$-porphyrin building block. In contrast, the $\mathbf{f} \mathbf{P} \mathbf{2}-\boldsymbol{Z} \cdot \mathbf{D} \mathbf{y}_{2}$ complex lacks the intramolecular inversion center, and the overall molecular anisotropy is thus the sum of the two non-collinear anisotropies at the $\mathrm{Dy}^{\mathrm{III}}$ sites. This key difference causes a stark difference in the observed torque. While $\boldsymbol{f}-\mathbf{P} 2-\boldsymbol{E} \cdot \mathrm{Dy}_{2}$ displays a periodic torque signal, centered around $\zeta=0$, the torque of $\boldsymbol{f} \mathbf{P 2}$ $Z \cdot \mathrm{Dy}_{2}$ complex is substantially shifted towards positive values. Physically, the former behavior is typical of a paramagnetic system while the latter is possible only for a blocked system that is allowed to reverse through tunneling at certain $\phi$. This is indeed shown by the permanent magnetization exhibited by the complex which shows tunneling effects at $20^{\circ}$ and $200^{\circ}$ (Figure 10). This indicates that the aromatic plane and the symmetry-breaking can influence dramatically the SMM behavior, leading to different quantum selection rules for tunneling.

In order to investigate the SMM behavior, we measured the dependence of the torque while sweeping $B$, for different orientations (Figure 11). Both compounds, $f-\mathrm{P} 2-\boldsymbol{E} \cdot \mathrm{Dy}_{2}$ and $f-\mathrm{P} 2-Z \cdot \mathrm{Dy}_{2}$, show the opening of a hysteresis cycle below $500 \mathrm{mK}$, with the cycles becoming wider at lower $T$, as is typical of the slow magnetization dynamics resulting from SMM behavior (Figure 11a,b). The hysteresis loop is considerably wider for $\boldsymbol{f}-\mathbf{P} 2-\boldsymbol{E} \cdot \mathbf{D y}_{2}$, although strong zero-field tunneling is still visible down to $50 \mathrm{mK}$. This is consistent with the presence of relatively strong Dy-Dy interactions in the compound, i.e. with a relaxation process that involves both Dy centers at the same time, and no spin-exchange bias at low field. The collinear anisotropy of $\boldsymbol{f}-\mathrm{P} 2-\boldsymbol{E} \cdot \mathrm{Dy}_{2}$ quenches tunneling of magnetic moment through transverse anisotropy terms that produces large hysteresis (Figure 11a). On the other hand, the noncollinear anisotropies of $\boldsymbol{f} \mathbf{P} \mathbf{2}-\boldsymbol{Z} \cdot \mathrm{Dy}_{2}$ allows overlapping of transverse anisotropy terms, and thereby increases tunneling probability, which decreases the observed hysteresis (Figure 11b). 

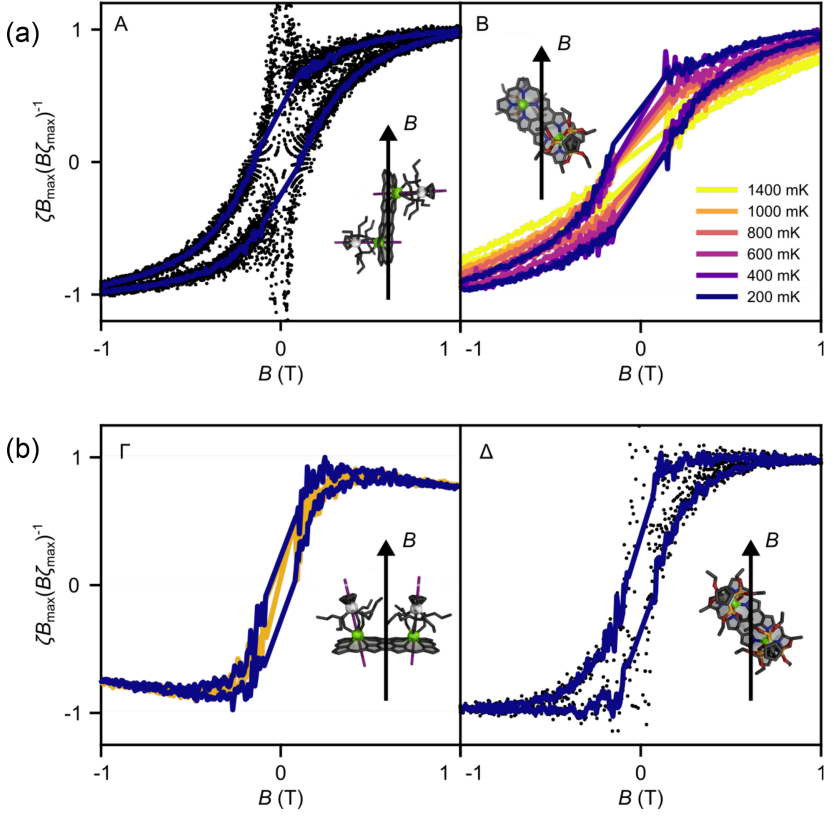

Figure 11. (a) Molecular hysteresis detected via torque magnetometry on single crystals of $\boldsymbol{f}-\mathbf{P} 2-E \cdot D y_{2}$. Black dots are measured data, solid lines the averaging over multiple $B$ sweeps. Symbols denote the orientations, as indicated in Figure 10. (b) Molecular hysteresis detected via torque magnetometry on single crystals of $\boldsymbol{f} \mathbf{P} \mathbf{2}-\boldsymbol{Z} \cdot \mathbf{D} \mathbf{y}_{2}$. Black dots are measured data, solid lines the averaging over multiple $B$ sweeps at different $T$ where blue lines are acquired at $0.2 \mathrm{~K}$. All measurements are acquired sweeping the external magnetic field $B$ at $0.125 \mathrm{~T} / \mathrm{min}$. Temperature is indicated in the color-scale, common to all panels. The direction of $B$ with respect to the molecular orientation are depicted in the insets. Symbols denote the orientations, as indicated in Figure 10.

\section{Conclusions}

The synthetic chemistry presented here establishes fused porphyrin oligomers bearing paramagnetic metal centers as prime compounds for investigating spin effects in $\pi$-conjugated nanostructures. Our results demonstrate it is possible to obtain a perfect definition of the $\pi$-plane-spin system: we reveal that lanthanide complexes of a singly-linked porphyrin dimer, $\boldsymbol{s}-\mathbf{P} \mathbf{2} \cdot \mathbf{L n}_{2}$, can be resolved into enantiomers, and that the corresponding complexes of an edge-fused porphyrin dimer can be separated into syn and anti diastereomers, $f-\mathbf{P 2}-Z \cdot \mathbf{L n}_{2}$ and $f-\mathbf{P} 2-E \cdot \mathbf{L n}_{2}$. In this way, the optical, electronic, electrochemical and magnetic properties of all the possible conformations of dinuclear $\mathrm{Dy}^{\mathrm{III}}$ and $\mathrm{Gd}^{\mathrm{III}}$ complexes can be compared and analyzed. The comparison between two types of dimers reveals that large differences in $\pi$-conjugation, which are strongly expressed in their absorption spectra and redox potentials, have less dramatic effects on the magnetic properties. Complete $\pi$ conjugation in the fused dimers results in comparatively strong magnetic exchange coupling between the metal centers. The static magnetic susceptibilities of the complexes at $300 \mathrm{~K}$ all match the expected values for isolated $\mathrm{Gd}^{\mathrm{III}}$ and $\mathrm{Dy}{ }^{\mathrm{III}}$ ions, whereas the lowtemperature magnetic susceptibility data reveal sizeable differences in the exchange coupling of the dimers. The precise determination of interactions using EPR yields syn couplings almost twice as large as those for the anti isomer $\left(J=-51 \pm 2 \mathrm{MHz}\right.$ in $f-\mathrm{P} 2-Z \cdot \mathbf{G d}_{2}$ and $J=$ $-19 \pm 3 \mathrm{MHz}$ in $f-\mathrm{P} 2-\boldsymbol{E} \cdot \mathrm{Gd}_{2}$ ), with vanishingly small exchange transmitted when conjugation is blocked in $s-\mathbf{P 2} \cdot \mathbf{G d}_{2}$.
The dynamic magnetic properties also reveal dramatic effects of the conformation around the $\pi$-conjugated plane. All the Dy ${ }^{I I I}$ complexes show similar activation behavior characteristic of a single molecule magnet. This confirms that the presence of a $\pi$ conjugated plane is not enough to perturb the large axial terms of the single-ion anisotropy of $\mathrm{Dy}^{\mathrm{III}}$. On the other hand, quantum tunneling and hysteresis cycles are much more sensitive to small perturbations of the transverse terms, as revealed by the torque in magnetic anisotropy at $50 \mathrm{mK}$. The difference in symmetry between syn and anti isomers in $\boldsymbol{f} \mathbf{P} \mathbf{2}-Z \cdot \mathbf{L n}_{2}$ and $\boldsymbol{f} \mathbf{P} \mathbf{2}-\boldsymbol{E} \cdot \mathbf{L n}_{2}$ leads to stark differences in the observed hysteresis, with narrower hysteresis and efficient tunneling in $f-\mathbf{P} 2-E \cdot \mathbf{L n}_{2}$.

The presence of a delocalized $\pi$-conjugated backbone is a primary component for electronic devices and for quantum processing. Our results reveal that the spin systems can interact via the $\pi$ conjugated backbone without losing the primary quantum properties, and $\mathrm{Gd}^{\mathrm{III}}$-based complexes can reach phase memory times up to $10 \mu \mathrm{s}$ at low temperatures. Such values are long enough to test quantum computing schemes using microwave pulses, and open up the path for information processing in single-molecule electronic devices. $^{44}$

Tunnelling in $\mathbf{f} \mathbf{P} 2-\boldsymbol{E} \cdot \mathrm{Dy}_{2}$ and $\mathbf{f} \mathbf{P} \mathbf{2}-\boldsymbol{Z} \cdot \mathrm{Dy}_{2}$ is visible at $\mathrm{mK}$ temperatures, and shows strong dependence on the syn vs. anti stereochemistry. These results confirm previous observation of strong environmental effects produced by delocalized $\pi$ states, for example in graphene, ${ }^{45}$ affording insights into SMMs on surfaces and carbon nanotubes, with geometrical discriminations that would otherwise not be available for spins grafted onto $\pi$-conjugated materials. This is a key step towards the creation of molecularly-tailored magnetic materials that are based on an aromatic plane and metal centers. Metalloporphyrin oligomers are an appealing family of compounds for the creation of aromatic materials with magnetic properties, because almost every metal in the periodic table can be inserted into a porphyrin. The current strategy can be extended to long multiple porphyrin chains, with up to 24 units for edge-fused tapes using published synthetic methods. ${ }^{13}$ There is also scope for extending this approach to nanorings that bear molecular magnets and support fully-delocalized electronic states. ${ }^{46}$ The observed interactions thus indicate encouraging perspectives for the use of these materials for multi-center quantum units. Their integration in poly-functional electronic devices, where the spin properties can be followed using single-molecule transport tools is also within reach.

\section{ASSOCIATED CONTENT}

\section{Supporting Information}

The Supporting Information is available free of charge at https://pubs.acs.org/doi/10.1021/jacsXXXXXXX.

Details of synthetic protocols, mass spectra and GPC traces, electrochemistry, magnetic characterization, EPR spectroscopy and torque magnetometry (PDF).

Crystallographic data for $\boldsymbol{s}$-P2 $\cdot \mathrm{Dy}_{2}(2154038)$ (CIF)

Crystallographic data for $s$-P2 $\cdot \mathbf{G d}_{2}(2154039)$ (CIF)

Crystallographic data for $\boldsymbol{f}$-P2- $\boldsymbol{Z} \cdot \mathbf{D y}_{2}(2154040)$ (CIF)

Crystallographic data for $\boldsymbol{f}-\mathrm{P} 2-\boldsymbol{E} \cdot \mathrm{Dy}_{2}(2154041)$ (CIF)

\section{AUTHOR INFORMATION}

\section{Corresponding Author}

${ }^{*}$ Harry L. Anderson - Department of Chemistry, University of Oxford, Chemistry Research Laboratory, Oxford OX1 3TA, United Kingdom; 
orcid.org/0000-0002-1801-8132;

Email: harry.anderson@chem.ox.ac.uk

* Lapo Bogani - Department of Materials, University of Oxford, Oxford, OX1 3PH, United Kingdom; orcid.org/0000-0002-4926-5048; Email:lapo.bogani@materials.ox.ac.uk

\section{Authors}

Jeff M. Van Raden - Department of Chemistry, University of Oxford, Chemistry Research Laboratory, Oxford OX1 3TA, United Kingdom; orcid.org/0000-0002-1801-8132;

Email: jeffrey.vanraden@chem.ox.ac.uk

Dimitris I. Alexandropoulos - Department of Materials, University of Oxford, Oxford OX1 3PH, United Kingdom; orcid.org/0000-00028759-9495; Email: Dimitrios.alexandropoulos@materials.ox.ac.uk Simen Sopp - Department of Materials, University of Oxford, Oxford OX1 3PH, United Kingdom; orcid.org/0000-0002-1200-7686; Email: simen.sopp@materials.ox.ac.uk

Michael Slota - Department of Materials, University of Oxford, Oxford OX1 3PH, United Kingdom; orcid.org/0000-0003-2979-5698

Email:michael.slota@materials.ox.ac.uk

Amber L. Thompson - Department of Chemistry, University of $O_{X-}$ ford, Chemistry Research Laboratory, Oxford OX1 3TA, United Kingdom; orcid.org/0000-0001-8258-860X

Email:amber.thompson@chem.ox.ac.uk

Taisuke Matsuno - Department of Chemistry, The University of Tokyo, Tokyo 113-0033, Japan; orcid.org/0000-0003-3887-5884 Email: tmatsuno@chem.s.u-tokyo.ac.jp

Hiroyuki Isobe - Department of Chemistry, The University of Tokyo, Tokyo 113-0033, Japan; orcid.org/0000-0001-8907-0694;

Email: isobe@chem.s.u-tokyo.ac.jp

\section{Author Contributions}

All authors have given approval to the final version of the manuscript. \$These authors contributed equally.

\section{ACKNOWLEDGMENTS}

We acknowledge financial support from the European Union (ERCCoG-773048-MMGNRs and ERC Advanced Grant 885606), the Royal Society (URF and grant), EPSRC (EP/N017188/1-QuEEN and $\mathrm{EP} / \mathrm{R} 042594 / 1)$.

\section{REFERENCES}

1. (a) De Volder, M. F. L.; Tawfick, S. H.; Baughman, R. H.; Hart, J. A. Carbon nanotubes: Present and future commercial applications Science 2013, 339, 535-539. (b) Castro Neto, A. H.; Guinea, F.; Peres, N. M. R.; Novoselov, K. S.; Geim, A. K. The electronic properties of graphene. Rev. Mod. Phys. 2009, 81, 109-162.

2. (a) Han, W.; Kawakami, R. K.; Gmitra, M.; Fabian, J. Graphene spintronics. Nat. Nanotechnol. 2014, 9, 794-807. (b) Choudhuri, I.; Bhauriyal, P.; Pathak, B. Recent advances in graphene-like 2D materials for spintronics applications. Chem. Mater. 2019, 31, 8260-8285.

3. Cervetti, C.; Rettori, A.; Pini, M. G.; Cornia, A.; Repollés, A.; Luis, F.; Dressel, M.; Rauschenbach, S.; Kern, K.; Burghard, M.; Bogani, L. The classical and quantum dynamics of molecular spins on graphene. Nat. Mater. 2016, 15, 164-168.

4. (a) Zoppellaro, G.; Bakandritsos, A.; Tuček, J.; Blońksi, P.; Susi, T.; Lazar, P.; Bad'ura, Z.; Steklý, T.; Opletalová, A.; Otyepka, M.; Zbořil, R. Microwave energy drives "on-off-on" spin-switch behavior in nitrogendoped graphene. Adv. Mater. 2019, 31, 1902587. (b) Langer, R.; Blońksi, P.; Hofer, C.; Lazar, P.; Mustonen, K.; Meyer, J. C.; Susi, T.; Otyepka, M. Tailoring electronic and magnetic properties of graphene by phosphorus doping. ACS Appl. Mater. Interfaces 2020, 12, 34074-34085. (c) Lin, L.; Fu, L.; Zhang, K.; Chen, J.; Zhang, W.; Tang, S.; Du, Y.; Tang, N. PSuperdoped graphene: Synthesis and magnetic properties. ACS Appl. Mater. Interfaces 2019, 11, 39062-39067. (d) Blońksi, P.; Tuček, J.; Sofer,
Z.; Mazánek, V.; Petr, M.; Pumera, M.; Otyepka, M.; Zbořil, R. Doping with graphitic nitrogen triggers ferromagnetism in graphene. J. Am. Chem. Soc. 2017, 139, 3171-3180. (e) Ito, Y.; Christodoulou, C.; Nardi, M. V.; Koch, N.; Kläui, M.; Sachdev, H.; Müllen, K. Tuning the magnetic properties of carbon by nitrogen doping of its graphene domains. J. Am. Chem. Soc. 2015, 137, 7678-7685.

5. Pesin, D; MacDonald, A. H. Spintronics and pseudospintronics in graphene and topological insulators. Nat. Mater. 2012, 11, 409-416.

6. Trauzettel, B.; Bulaev, D. V.; Loss, D.; Burkard, G. Spin qubits in graphene quantum dots. Nat. Phys. 2007, 3, 192-196.

7. (a) Narita, A.; Wang, X.-Y.; Feng, K.; Müllen, K. New advances in nanographene chemistry. Chem. Soc. Rev. 2015, 44, 6616-6643. (b) Wang, X.-Y.; Yao, X.; Narita, A.; Müllen, K. Heteroatom-doped nanographenes with structural precision. Acc. Chem. Res. 2019, 52, 2491-2505. (c) Slota, M.; Keerthi, A.; Myers, W. K.; Tretyakov, E.; Baumgarten, M.; Ardavan, A.; Sadeghi, H.; Lambert, C. J.; Narita, A.; Müllen, K.; Bogani, L. Magnetic edge states and coherent manipulation of graphene nanoribbons. Nature 2018, 557, 691-695. (d) Rizzo, D. J.; Veber, G.; Cao, T.; Bronner, C.; Chen, T.; Zhao, F.; Rodriguez, H.; Louie, S. G.; Crommie, M. F.; Fischer, F. R. Topological band engineering of graphene nanoribbons. Nature 2018, 560, 204-208.

8. Itami, K.; Maekawa, T. Molecular nanocarbon science: Present and future. Nano Lett. 2020, 20, 4718-4720.

9. Ishikawa, N.; Sugita, M.; Ishikawa, T.; Koshihara, S. Y.; Kaizu, Y. Lanthanide double-decker complexes functioning as magnets at the singlemolecular level. J. Am. Chem. Soc. 2003, 125, 8694-8695.

10. (a) Woodrudd, D.; Winpenny, R. E. P.; Layfield, R. A. Lanthanide single-molecule magnets. Chem. Soc. Rev. 2013, 113, 5110-5148. (b) Marin, R.; Brunet, G.; Murugesu, M. Shining new light on multifunctional lanthanide single-molecule magnets. Angew. Chem. Int. Ed. 2019, 60, 1728-1746. (c) Goodwin, C. A. P.; Ortu, F.; Reta, D.; Chilton, N. F.; Mills, D. P. Molecular magnetic hysteresis and 60 kelvin in dysprosocenium. Nature 2017, 548, 439-442. (d) Guo, F. S.; Day, B. M.; Chen, Y. C.; Tong, M. L.; Mansikkamaki, A.; Layfield, R. A. Magnetic hysteresis up to 80 kelvin in a dysproium metallocene single-molecule magnet. Science 2018, 362, 1400-1403. (e) Coronado, E. Molecular magnetism: from chemical design to spin control in molecules, materials and devices. Nat. Rev. Mater. 2020, $5,87-104$.

11. Le Roy, J. L.; Cremers, J.; Thomlinson, I. A.; Slota, M.; Myers, W. K.; Horton, P. H.; Coles, S. J.; Anderson, H. L.; Bogani, L. Tailored homo- and hetero-lanthanide porphyrin dimers: a synthetic strategy for integrating multiple spintronic functionalities into a single molecule. Chem. Sci. 2018, 9, 8474-8481.

12. (a) Osuka, A.; Shimidzu, H. meso,meso-Linked porphyrin arrays. Angew. Chem. Int. Ed. Engl. 1997, 36, 135-137. (b) Aratani, N.; Osuka, A.; Kim, Y. H.; Jeong, D. H. J.; Kim, D. Extremely long, discrete meso-meso coupled porphyrin arrays. Angew. Chem. Int. Ed. Engl. 2000, 39, 14581462. (c) Aratani, N.; Takagi, A.; Yanagawa, Y.; Matsumoto, T.; Kawai, T.; Yoon, Z. S.; Kim, D.; Osuka, A. Giant meso-meso-linked porphyrin arrays of micrometer molecular length and their fabrication. Chem. Eur. J. 2005, $11,3389-3404$

13. (a) Tsuda, A.; Furuta, H.; Osuka, A. Completely Fused Diporphyrins and Triporphyrins. Angew. Chem. Int. Ed. 2000, 39, 2549-2552. (b) Tsuda, A.; Furuta, H.; Osuka, A. Syntheses, structural characterizations, and optical and electrochemical properties of directly fused diporphyrins. $J$. Am. Chem. Soc. 2001, 123, 10304-10321. (c) Tsuda, A.; Osuka, A. Fully conjugated porphyrin tapes with electronic absorption bands that reach into infrared. Science 2001, 293, 79-82. (d) Cho, H. S.; Jeong, D. H.; Cho, S.; Kim, D.; Matsuzaki, Y.; Tanaka, K.; Tsuda, A.; Osuka, A. Photophysical properties of porphyrin tapes. J. Am. Chem. Soc. 2002, 124, 14642-14654. (e) Ikeda, T.; Aratani, N.; Osuka, A. Synthesis of extremely $\pi$-extended porphyrin tapes from hybrid meso-meso linked porphyrin arrays: An approach towards the conjugation length. Chem. Asian J. 2009, 4, 1248 1256. (f) Tanaka, A.; Osuka, A. Conjugated porphyrin arrays: synthesis, properties and applications for functional materials. Chem. Soc. Rev. 2015, 44, 943-969. (g) Tanaka, T.; Osuka, A. Triply linked porphyrinoids. Chem. Eur. J. 2018, 24, 17188-17200. 
14. (a) Park, M.; Cho, S.; Yoon, Z. S.; Aratani, N.; Osuka, A.; Kim, D. Single molecule spectroscopic investigation on conformational heterogeneity of directly linked zinc(II) porphyrin arrays. J. Am. Chem. Soc. 2005 127, 15201-15206. (b) Yang, J.; Yoo, H.; Aratani, N.; Osuka, A.; Kim, D. Determination of the superradiance coherence length of directly linked linear porphyrin arrays at the single-molecule level. Angew. Chem. Int. Ed. 2009, 48, 4323-4327.

15. (a) Yamaguchi, Y. Time-dependent density functional calculations of fully $\pi$-conjugated zinc oligoporphyrins J. Chem. Phys. 2002, 117, 9688 9694. (b) Pedersen, T. G.; Lynge, T. B.; Kristensen, P. K.; Johansen, P. M. Theoretical study of conjugated porphyrin polymers. Thin Solid Films 2005, 477, 182-186. (c) Ohmori, S.; Kawabata, H.; Tokunaga, K.; Tachikawa, H. Molecular design of high performance fused porphyrin onedimensional wire: A DFT study. Thin Solid Films 2009, 518, 901-905. (d) Posligua, V.; Aziz, A.; Haver, R.; Peeks, M. D.; Anderson, H. L.; GrauCrespo, R. Band structures of periodic porphyrin nanostructures. J. Phys Chem. C 2018, 122, 23790-23798.

16. (a) Liu, H.; Yu, C.; Gao, N.; Zhao. J. The diversity of electrontransport behaviors of molecular junctions: Correlation with the electrontransport pathway. ChemPhysChem 2010, 11, 1895-1902. (b) Sedghi, G.; Esdaile, L. J.; Anderson, H. L.; Martin, S.; Bethell, D.; Higgins, S. J.; Nichols, R. J. Comparison of the conductance of three types of porphyrin-based molecular wires: $\beta$,meso, $\beta$-fused tapes, meso-butadiyne-linked and twisted meso-meso linked oligomers. Adv. Mater. 2012, 24, 653-657. (c) Algethami, N.; Sadeghi, H.; Sangtarash, S.; Lambert, C. J. The conductance of porphyrin-based molecular nanowires increases with length. Nano Lett. 2018, 18, 4482-4486. (d) Leary, E.; Limburg, B.; Alanazy, A.; Sangtarash, S.; Grace, I.; Swada, K.; Esdaile, L. J.; Noori, M.; González, M. T.; RubioBollinger, G.; Sadeghi, H.; Hodgson, A.; Agrait, N.; Higgins, S. J.; Lambert, C. J.; Anderson, H. L.; Nichols, R. J. Bias-driven conductance increase with length in porphyrin tapes. J. Am. Chem. Soc. 2018, 140, 12877-12883.

17. Lee, S.; Yamashita, K. I.; Sakata, N.; Hirao, Y.; Ogawa, K.; Ogawa, T. Stable singlet biradicals of rare-earth-fused diporphyrin-triple-decker complexes with low energy gaps and multi-redox states. Chem. Eur. J. 2019, 25, 3240-3243.

18. (a) Ikeue, T.; Furukawa, K.; Hata, H.; Aratani, N.; Shinokubo, H.; Kato T.; Osuka, A. The importance of a $\beta-\beta$ bond for long-range antiferromagnetic coupling in directly linked copper(II) and silver(II) diporphyrins. Angew. Chem. Int. Ed. 2005, 44, 6899-6901. (b) Wili, N.; Richert, S.; Limburg, B.; Clarke, S. J.; Anderson, H. L.; Timmel, C. R.; Jeschke, G. ELDOR-detected NMR beyond hyperfine couplings: a case study with Cu(II)-porphyrin dimers. Phys. Chem. Chem. Phys. 2019, 21, $11676-$ 11688 .

19. Kläui, W. The coordination chemistry and organometallic chemistry of tridentate oxygen ligands with $\pi$-donor properties. Angew. Chem. Int. Ed. 1990, 29, 627-637

20. (a) Gao, F.; Yao, M.-X.; Li, Y.-Y.; Li, Y.-Z.; Song, Y.; Zuo, J.-L. Syntheses, structures, and magnetic properties of seven-coordinate lanthanide porphyrinate or phthalocyaninate complexes with Kläui's tripodal ligand. Inorg. Chem. 2013, 52, 6407-6416. (b) Chan, W.-L.; Xie, C.; Lo, W.-S.; Bünzli, J.-C. G.; Wong, W.-K.; Wong, K.-L. Lanthanide-tetrapyrrole complexes: synthesis, redox chemistry, photophysical properties, and photonic applications. Chem. Soc. Rev. 2021, 50, 12189-12257.

21. Kaolta, B.; Mikus, A.; Ostrowski, S. Synthesis of lutetium(III)porphyrin complexes: Old problems and new excellent conditions found. New J. Chem. 2016, 40, 9899-9902.

22. Ouyang, Q.; Zhu, Y. Z.; Zhang, C. H.; Yan, K. Q.; Li, Y. C.; Zheng, J. Y. An efficient PIFA-mediated synthesis of fused diporphyrin and triplysingly interlacedly linked porphyrin array. Org. Lett. 2009, 11, 5266-5269.

23. Single crystal X-ray diffraction data were collected using a (Rigaku) Oxford Diffraction SuperNova A diffractometer and reduced using CrysAlisPro. Structures were solved using SuperFlip [Palatinus, L.; Chapuis, G. SUPERFLIP - a computer program for the solution of crystal structures by charge flipping in arbitrary dimensions. J. Appl. Cryst. 2007, 40, 786-790] and refined using CRYSTALS [Betteridge, P. W.; Carruthers, J. R.; Cooper, R. I.; Prout, K.; Watkin, D. J. CRYSTALS version 12: software for guided crystal structure analysis. J. Appl. Cryst. 2003, 36, 1487; Cooper, R. I.;
Thompson, A. L.; Watkin, D. J. CRYSTALS enhancements: dealing with hydrogen atoms in refinement. J. Appl. Cryst. 2010, 43, 1100-1107] as per the SI (CIF). Non-bonded distances and angles were calculated using PLATON [Spek, A. L. Single-crystal structure validation with the program PLATON. J. Appl. Cryst. 2003, 36, 7-13].

24. Dime, A. K. D.; Devillers, C. H.; Cattey, H.; Habermeyer, B.; Lucas, D. Control over the oxidative reactivity of metalloporphyrins. Efficient electrosynthesis of meso,meso-linked zinc porphyrin dimer. Dalton Trans. 2012, 41, 929-936.

25. (a) McEwan, K. J.; Fleitz, P. A.; Rogers, J. E.; Slagle, J. E.; McLean, D. G.; Akdas, H.; Katterle, M.; Blake, I. M.; Anderson, H. L. Reverse saturable absorption in the near-infrared by fused porphyrin dimers. Adv. Mater. 2004, 16, 1933-1935. (b) Tanaka, T.; Lee, B. S.; Aratani, N.; Yoon, M.-C.; Kim, D.; Osuka, A. Synthesis and properties of hybrid porphyrin tapes. Chem. Eur. J. 2011, 17, 14400-14412.

26. Yoshida, N.; Ishizuka, T.; Osuka, A.; Jeong, D. H.; Cho, H. S.; Kim, D.; Matsuzaki, Y.; Nogami, A.; Tanaka, K. Fine tuning of photophysical properties of meso-meso-linked $\mathrm{Zn}^{\mathrm{II}}$-diporphyrins by dihedral angle control. Chem. Eur. J. 2002, 9, 58-75.

27. Shinmori, H.; Ahn, T. K.; Cho, H. S.; Kim, D.; Yoshida, N.; Osuka, A. Dihedral-angle modulation of meso-meso-linked $\mathrm{Zn}^{\mathrm{II}}$ diporphyrin through diamine coordination and its application to reversible switching of excitation energy transfer. Angew. Chem. Int. Ed. 2003, 42, 2754-2758.

28. Ooi, S.; Shimizu, D.; Furukawa, K.; Tanaka, T.; Osuka, A. Stable face-to-face singlet diradicaloids: Triply linked corrole dimer gallium(III) complexes with two $\mu$-hydroxo bridges. Angew. Chem. Int. Ed. 2018, 57, $14916-14920$.

29. Shi, J. H.; Li, Z.; Liu, W. Y.; Yu, M.; Li, Z. H.; Liu, G. F. Synthesis and properties study of asymmetrical carbazole porphyrin with $p$ hydroxylphenyl and its metal complexes (Zn, Dy). Synth. React. Inorg. Nano-Met. Chem. 2013, 43, 316-320.

30. (a) Atzori, M.; Rikken, G. L. J. A.; Train, C. Magneto-chiral dichroism: A playground for molecular chemists. Chem. Eur. J. 2020, 26, 9784 9791. (b) Train, C.; Grusellec, M.; Verdaguer, M. The fruitful introduction of chirality and control of absolute configurations in molecular magnets. Chem. Soc. Rev. 2011, 40, 3297-3312.

31. Yoshida, N.; Osuka, A. First optical resolution of meso-meso linked diporphyrin. Tetrahedron Lett. 2000, 41, 9287-9291.

32. Ouyang, Q.; Zhu, Y. Z.; Li, Y. C.; Wei, H. B.; Zheng, J. Y. Diastereoselective synthesis of chiral diporphyrins via intramolecular mesomeso oxidative coupling. J. Org. Chem. 2009, 74, 3164-3167.

33. Gehrold, A. C.; Bruhn, T.; Bringham, G. Axial, helical, and planar chirality in directly linked basket-handle porphyrin arrays. J. Org. Chem. 2016, 81, 1075-1088.

34. (a) Brodbeck, C. M.; Iton, L. E. The EPR spectra of $\mathrm{Gd}^{3+}$ and $\mathrm{Eu}^{2+}$ in glassy systems. J. Chem. Phys. 1985, 83, 4285-4299. (b) Spyroulias, G. A.; Coutsolelos, A. G. Synthesis and spectroscopic studies of lanthanide monoporphyrinates with meso-tetra-aryl porphyrinate bearing bromines on the $\beta$-pyrrole positions of fluorines on phenyl groups. Polyhedron 1995, 14, 2483-2490. (c) Spyroulias, G. A.; Raptopoulou, C. P.; de Montauzon, D.; Mari, A.; Poilblanc, R.; Terzis, A.; Coutsolelos, A. G. Synthesis and physicochemical characterization of protonated and deprotonated forms in heteroleptic lanthanide(III) porphyrinate double-deckers. X-ray structure of $\mathrm{Gd}^{\mathrm{III}} \mathrm{H}$ (oep) (tpp) at 298 and 21 K. Inorg. Chem. 1999, 38, 1683-1696.

35. Dalaloyan, A.; Qi, M.; Ruthstein, S.; Vega, S.; Godt, A.; Feintuch, A.; Goldfarb, D. Gd(III)-Gd(III) EPR distance measurements - the range of accessible distances and the impact of zero field splitting Phys. Chem. Chem. Phys. 2015, 17, 18464-18476.

36. Eaton, G. R.; Eaton, S. S. in Multifrequency Electron Paramagnetic Resonance: Theory and Applications (ed. Misra, S. K.) Ch. 17, Wiley, Weinheim, 2011.

37. Hermenau, J.; Ibañez-Azpiroz, J.; Hubner, C.; Sonntag, A.; Baxevanis, B.; Ton, K. T.; Steinbrecher, M.; Khajetoorians, A. A.; dos Santos Dias, M.; Blügel, S.; Wiesendanger, R.; Lounis, S.; Wiebe, J. A gateway towards non-collinear spin processing using three-atom magnets with strong substrate coupling. Nat. Commun. 2017, 8, 642. 
38. Benelli, C.; Gatteschi, D. Magnetism of lanthanides in molecular materials with transition-metal ions and organic radicals. Chem. Rev. 2002, 102, 2369-2388

39. Jiang, S.-D.; Wang, B.-W.; Su, G.; Wang, Z.-M.; Gao, S. A mononuclear dysprosium complex featuring single-molecule-magnet behavior. Angew. Chem. Int. Ed. 2010, 49, 7448-7451.

40. Borilovic, I.; Alonso, P. J.; Roubeau, O.; Aromí, G. A bis-vanadyl coordination complex as a 2-qubit quantum gate. Chem. Commun. 2020, 56, 3139-3142.

41. Bagai, R.; Christou, G. The Drosophila of single-molecule magnetism: $\left[\mathrm{Mn}_{12} \mathrm{O}_{12}\left(\mathrm{O}_{2} \mathrm{CR}\right)_{16}\left(\mathrm{H}_{2} \mathrm{O}\right)_{4}\right]$. Chem. Soc. Rev. 2009, 38, $1011-$ 1026.

42. (a) Gu, L.; Wu, R. Origin of the anomalously low Raman exponents in single molecule magnets. Phys. Rev. B 2021, 103, 014401. (b) Liddle, S. T.; van Slageren, J. Improving f-element single molecule magnets. Chem. Soc. Rev. 2015, 44, 6655-6669.

43. (a) Singh, A.; Shrivastava, K. N. Opto-acoustic two-photon relaxation in spin systems. Phys. Status Solidi B 1979, 95, 273-277. (b) Novikov, V. V.; Pavlov, A. A.; Nelyubina, Y. V.; Boulon, M.-E.; Varzatskii, O. A.; Voloshin; Y. Z.; Winpenny, R. E. P. A trigonal prismatic mononuclear cobalt(II) complex showing single-molecule magnet behavior. J. Am. Chem. Soc. 2015, 137, 9792-9795.

44. (a) Urdampilleta, M.; Klyatskaya, S.; Cleuziou, J.-P.; Ruben, M.; Wernsdorfer, W. Supramolecular spin valves. Nat. Mater. 2011, 10, 502 506. (b) Bogani, L.; Wernsdorfer, W. Molecular spintronics using singlemolecule magnets. Nat. Mater. 2008, 7, 179-186. (c) Gehring, P.; Sowa, J. K.; Hsu, C.; de Bruijckere, J.; van der Star, M.; Le Roy, J. J.; Bogani, L.; Gauger, E. M.; van der Zant, H. S. Complete mapping of the thermoelectric properties of a single molecule. Nat. Nanotechnol. 2021,16, 426-430. (d) Vincent, R.; Klyatskaya, S.; Ruben, M.; Wernsdorfer, W.; Balestro, F. Electronic read-out of a single nuclear spin using a molecular spin transistor. Nature 2012, 488, 357-359. (e) Burzurí, E.; Zyazin, A. S.; Cornia, A.; van der Zant, H. S. J. Direct observation of magnetic anisotropy in an individual $\mathrm{Fe}_{4}$ single-molecule magnet. Phys. Rev. Lett. 2012, 109, 147203 (f) Aragonès, A. C.; Aravena, D.; Cerdá, J. I.; Acís-Castillo, Z.; Li, H.; Real, J. A.; Sanz, F.; Hihath, J.; Ruiz, E.; Díez-Pérez, I. Large conductance switching in a single-molecule device through room temperature spin-dependent transport. Nano Lett. 2016, 16, 218-226.

45. (a) Sakurai, M.; Koley, P.; Aono, M. Tunable magnetism of organometallic nanoclusters by graphene oxide on-surface chemistry. $S_{c i}$. Rep. 2019, 9, 14509. (b) Paschke, F.; Erler, P.; Enenkel, V.; Gragnaniello, L.; Fonin, M. Bulk-like magnetic signature of individual $\mathrm{Fe}_{4} \mathrm{H}$ molecular magnets on graphene. ACS Nano 2019, 13, 780-785. c) Spree, L.; Liu, F.; Neu, V.; Rosenkranz, M.; Velkos, G.; Wang, Y.; Schiemenz, S.; Dreiser, J.; Gargiani, P.; Valvidares, M.; Chen, C.-H.; Bücher, B.; Avdoshenko, S. M.; Popov, A. A. Robust single molecule magnet monolayers on graphene and graphite with magnetic hysteresis up to $28 \mathrm{~K}$. Adv. Funct. Mater. 2021, 31, 2105516. (d) Baltic, R.; Pivetta, M.; Donati, F.; Wäckerlin, C.; Singha, A.; Dreiser, J.; Rusponi, S.; Brune, H. Superlattice of single atom magnets on graphene. Nano Lett. 2016, 16, 7610-7615. (e) Baltic, R.; Donati, F.; Singha, A.; Wäckerlin, C.; Dreiser, J.; Delley, B.; Pivetta, M.; Rusponi, S.; Brune, H. Magnetic properties of single rare-earth atoms on graphene/Ir(111). Phys. Rev. B 2018, 98, 024412.

46. (a) Peeks, M. D.; Claridge, T. D. W.; Anderson, H. L. Aromatic and antiaromatic ring currents in a molecular nanoring, Nature 2017, 541, 200 203. (b) Rickhaus, M.; Jirasek, M.; Tejerina, L.; Gotfredsen, H.; Peeks, M. D.; Haver, R.; Jiang, H.-W.; Claridge, T. D. W.; Anderson, H. L. Global aromaticity at the nanoscale. Nat. Chem. 2020, 12, 236-241. 
Graphic entry for the Table of Contents

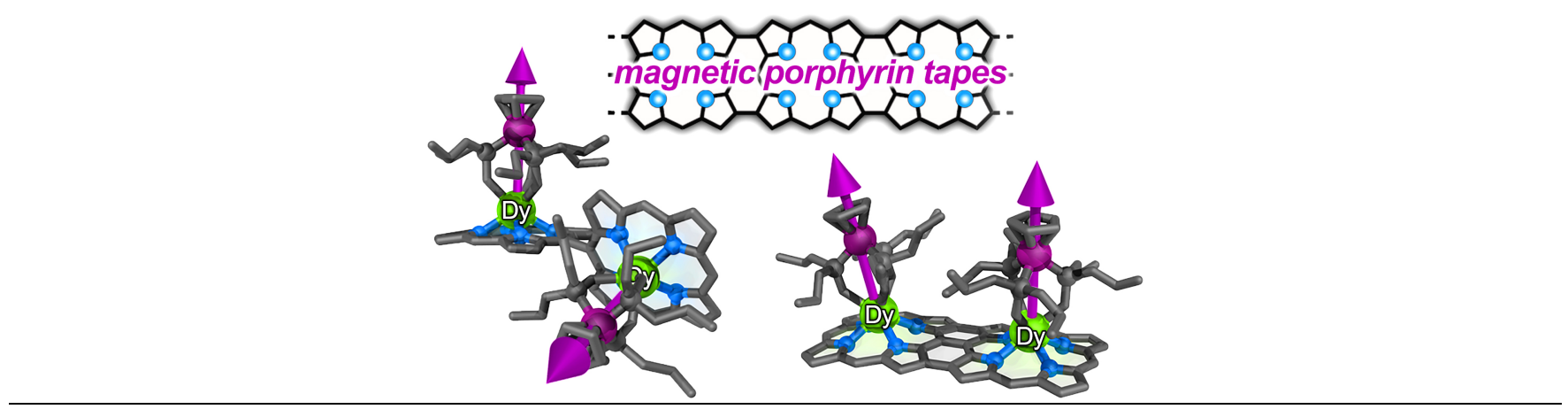

14 